\title{
How the $\mathrm{O}_{2}$-dependent $\mathrm{Mg}$-protoporphyrin monomethyl ester cyclase forms the fifth ring of chlorophylls
}

\author{
Guangyu E. Chen ${ }^{\mathrm{a}, 1,2}$, Nathan B. P. Adams ${ }^{\mathrm{a}}$, Philip J. Jackson ${ }^{\mathrm{a}, \mathrm{b}}$, Mark J. Dickman ${ }^{\mathrm{b}}$, C. Neil \\ Hunter ${ }^{\mathrm{a}, 2}$ \\ aDepartment of Molecular Biology and Biotechnology, University of Sheffield, Sheffield S10 2TN, \\ UK \\ ${ }^{b}$ Department of Chemical and Biological Engineering, University of Sheffield, Sheffield S1 3JD, \\ UK
}

\section{Abstract}

Mg-protoporphyrin IX monomethyl ester (MgPME) cyclase catalyses the formation of the isocyclic ring, the hallmark of chlorins and bacteriochlorins, producing protochlorophyllide $a$ and contributing significantly to the absorption properties of chlorophylls and bacteriochlorophylls. The $\mathrm{O}_{2}$-dependent cyclase is found in oxygenic phototrophs and in some purple bacteria. We overproduced the simplest form of the cyclase, AcsF from Rubrivivax gelatinosus, in Escherichia coli. In biochemical assays the diiron cluster within AcsF is reduced by ferredoxin furnished by NADPH and ferredoxin:NADP ${ }^{+}$reductase or by direct coupling to Photosystem I photochemistry, linking cyclase to the photosynthetic electron transport chain. Kinetic analyses yield a $k_{\text {cat }}$ of 0.9 $\min ^{-1}$, a $K_{\mathrm{M}}$ of $7.0 \mu \mathrm{M}$ for MgPME, and a $K_{\mathrm{d}}$ for MgPME of $0.16 \mu \mathrm{M}$. Mass spectrometry identified $13^{1}$-hydroxy-MgPME and $13^{1}$-keto-MgPME as intermediates in the formation of the isocyclic ring, revealing the reaction chemistry that converts porphyrins to chlorins, and completing the work originated by Sam Granick in 1950.

Chlorophylls, a class of cyclic tetrapyrroles, are among the most abundant natural pigments on Earth. They are the major absorbers of the solar energy that drives photosynthesis, and billions of tonnes of chlorophyll are synthesised annually on land and in the oceans. The decisive biosynthetic step that determines the absorption properties of chlorophyll, and more visually its green color, is the formation of the unique isocyclic fifth ring. This process involves the conversion of Mg-protoporphyrin IX monomethyl ester (MgPME) to 3,8divinyl protochlorophyllide $a$ (DV PChlide a), and it requires incorporation of an oxygen atom, sourced from either water or $\mathrm{O}_{2}$ (refs. ${ }^{1,2}$ ), indicating the existence of two

\footnotetext{
Users may view, print, copy, and download text and data-mine the content in such documents, for the purposes of academic research, subject always to the full Conditions of use:http://www.nature.com/authors/editorial_policies/license.html\#terms

${ }^{2}$ To whom correspondence should be addressed. guangyu.chen@sjtu.edu.cn; c.n.hunter@sheffield.ac.uk.

${ }^{1}$ Current address: School of Life Sciences and Biotechnology, Shanghai Jiao Tong University, Shanghai 200240, China

Author contributions

G.E.C., N.B.P.A., P.J.J., M.J.D. and C.N.H. designed the research. G.E.C., N.B.P.A. and P.J.J. performed research and analysed data G.E.C., N.B.P.A., P.J.J., M.J.D. and C.N.H. wrote the manuscript.

Competing interests

The authors declare no competing interests.
} 
mechanistically different MgPME cyclases. Most anoxygenic phototrophic bacteria utilise an $\mathrm{O}_{2}$-sensitive radical SAM enzyme containing [4Fe-4S] and cobalamin cofactors to catalyse the reaction ${ }^{3}$, while oxygenic phototrophs including cyanobacteria, algae and plants, as well as some purple bacteria, adopt an $\mathrm{O}_{2}$-dependent cyclase for the reaction. Three classes of $\mathrm{O}_{2}$-dependent cyclase have been identified ${ }^{4}$, all with a catalytic subunit AcsF, a putative diiron protein ${ }^{5}$, but they differ in the requirement for an auxiliary subunit, either Ycf54 for the enzyme found in oxygenic phototrophs ${ }^{6,7}$, or BciE for the alphaproteobacterial enzyme ${ }^{4}$. However, there have been no mechanistic studies of the $\mathrm{O}_{2}$ dependent cyclase using purified components, so the details of this important reaction have remained unknown since Granick ${ }^{8}$ proposed a sequence of reactions that form the isocyclic fifth or E ring.

Early biochemical characterisation of cyclase activity using either intact or fractionated plant developing chloroplasts demonstrated the necessity for $\mathrm{O}_{2}$ (refs. ${ }^{9-12}$ ), and inhibitor studies with specific chelators showed that iron is also required ${ }^{11}$. Both NADPH and NADH were found to stimulate cyclase activity, with NADPH more effective than NADH (refs. ${ }^{11,13}$ ). These properties are shared by the enzymes from the green alga Chlamydomonas $(C$.) reinhardtii and the cyanobacterium Synechocystis sp. PCC 6803 (hereafter Synechocystis) ${ }^{14,15}$ and are characteristic of iron-dependent oxygenases. In addition, the cucumber, Synechocystis and barley enzymes were resolved into soluble and membranebound components ${ }^{13,15,16}$, but it has not been possible to obtain an active, pure cyclase from a native source.

The complexity of $\mathrm{E}$ ring formation implies the involvement of multiple sequential reactions. Based on the mechanism of $\beta$-oxidation of fatty acids, Granick ${ }^{8}$ proposed the reaction could proceed through $\beta$-oxidation of the $\mathrm{C} 13$ methylpropionyl group of MgPME, via $13^{1}-13^{2}$ acrylate, $13^{1}$-hydroxy and $13^{1}$-keto intermediates. These proposed reaction intermediates were subsequently detected in some Chlorella mutants ${ }^{17,18}$, and Castelfranco and co-workers confirmed the $13^{1}$-hydroxy and $13^{1}$-keto intermediates using the reconstituted cucumber cyclase system ${ }^{19}$. The $13^{1}$-hydroxy derivative of MgPME was detected in these assays and shown to be an active cyclase substrate, and a similar compound was also identified during measurements of cyclase activity in isolated chloroplasts from $C$. reinhardtii (ref. ${ }^{14}$ ). A chemically synthesised $13^{1}$-keto analog of MgPME is readily converted to the final cyclase product, thereby validating this intermediate in the reaction sequence ${ }^{19}$. However, a synthesised acrylate derivative of MgPME was found to be inactive as a cyclase substrate in the reconstituted cyclase system ${ }^{20}$. It is possible that the acrylate derivative detected in the Chlorella mutants resulted from the $13^{1}$-hydroxy intermediate undergoing a reverse hydratase reaction ${ }^{1}$. Based on these findings, the original reaction scheme of Granick and others has been modified to omit the acrylate intermediate, as shown in Fig. 1.

Elucidation of the cyclase reaction sequence requires quantities of pure, active enzyme sufficient for biochemical and kinetic analyses. Although there have been no reports of the purification and in vitro reconstitution of the $\mathrm{O}_{2}$-dependent cyclase, an active recombinant cyclase was used successfully as part of a complete ensemble of biosynthetic enzymes that collectively enabled E. coli to synthesise chlorophyll ${ }^{21}$. Here we report the purification of 
the single-subunit $\mathrm{O}_{2}$-dependent cyclase AcsF from Rubrivivax (Rvi.) gelatinosus (ref. ${ }^{5}$ ). Cyclase activity was reconstituted using a tricomponent electron transfer system consisting of NADPH, ferredoxin (Fd) and Fd:NADP ${ }^{+}$reductase (FNR), and the steady-state kinetic behaviour of the enzyme was characterised. Furthermore, we detected the two previously proposed reaction intermediates in the progress of the reaction and subsequently determined their chemical identity by mass spectrometry. Our work therefore lays the groundwork for future mechanistic and structural study of the $\mathrm{O}_{2}$-dependent cyclase involved in chlorophyll biosynthesis.

\section{Purification of AcsF from Rvi. gelatinosus}

Rvi. gelatinosus AcsF with an $\mathrm{N}$-terminal $\mathrm{His}_{6}$ tag was produced in the E. coli $\mathrm{C} 43$ (DE3) strain $^{22}$, supplemented with ferric ammonium citrate (see Materials and Methods). Histagged AcsF was purified by Ni-IDA affinity chromatography from membrane fractions solubilised with the non-ionic detergent $n$-dodecyl- $\beta$-D-maltoside ( $\beta$-DDM), followed by an iron reconstitution step with ferrous ammonium sulphate to increase the occupancy of the iron-binding sites. Subsequent gel filtration chromatography, in which a single symmetrical elution peak was observed (Fig. 2a), purified AcsF further. SDS-polyacrylamide gel electrophoresis (PAGE) analysis shows a single polypeptide with an apparent molecular mass of $\sim 37 \mathrm{kDa}$ (Fig. 2a; inset), and migrating further through the gel than expected for a predicted mass of $44 \mathrm{kDa}$, a common feature of membrane-associated proteins. The protein was over $95 \%$ pure, with an overall yield of $\sim 3 \mathrm{mg}$ per litre of culture.

To investigate the oligomerisation state of the purified AcsF protein, gel filtration calibration curves were produced by analysing commercially available soluble protein standards, and four membrane protein standards available in our laboratory (Fig. 2b). We assumed that the collective mass of $\beta$-DDM molecules in complex with membrane proteins is the same as a $\beta$-DDM micelle, which is $72 \pm 1.4 \mathrm{kDa}$ (ref. ${ }^{23}$ ). The purified AcsF with the associated $\beta$ DDM molecules was estimated to have a molecular mass of $\sim 210 \mathrm{kDa}$ with soluble standards, and $\sim 180 \mathrm{kDa}$ using membrane protein standards (Fig. 2b). By subtracting the contribution from detergent molecules, this indicates that AcsF is dimeric or trimeric given the predicted molecular mass of $44 \mathrm{kDa}$ from the primary sequence.

\section{Spectroscopic and biophysical characterisation of AcsF}

Purified AcsF has a pale brown colour and exhibits a broad and weak band centred at $\sim 340$ $\mathrm{nm}$ in the absorbance spectrum (Fig. 2c), which is from an oxo-to-Fe(III) charge transfer transition ${ }^{24}$. An additional band at $\sim 410 \mathrm{~nm}$ was also observed (Fig. 2c). Upon addition of 2 $M$ sodium azide, formation of a chromophore with broad absorbance bands at 345 and 450 $\mathrm{nm}$ was observed and the position of the $\sim 410 \mathrm{~nm}$ band was unaffected (Fig. $2 \mathrm{~d}$ ). The optical features, including the $\sim 340 \mathrm{~nm}$ charge transfer band and the 345 and $450 \mathrm{~nm}$ bands when in complex with azide, are characteristic of $\mu$-oxo-bridged diiron clusters, which have been reported with other diiron proteins such as stearoyl-acyl carrier protein $\Delta^{9}$ desaturase ${ }^{24}$, $\mathrm{CmlA}$ in chloramphenicol biosynthesis ${ }^{25}$, and CLK-1 in ubiquinone biosynthesis ${ }^{26}$. Assays determined that AcsF contained $2.35 \pm 0.04$ iron per monomer (Supplementary Fig. 1) providing further evidence for the presence of a diiron cluster in AcsF. We used differential 
scanning calorimetry to analyse the thermostability of AcsF and the melting point was determined to be $57.2^{\circ} \mathrm{C}$ (see Supplementary Fig. 2 for the melting curve), indicating the protein is stable for activity tests at $30^{\circ} \mathrm{C}$.

\section{In vitro reconstitution of cyclase activity with AcsF}

To test whether the purified AcsF is active, we conducted end-point in vitro assays, followed by pigment analysis using high performance liquid chromatography (HPLC). Apart from the porphyrin substrate, MgPME, and molecular oxygen, an electron donor is also required to reduce the diiron center of $\mathrm{AcsF}$ from +3 to +2 during the catalytic cycle as required by most other diiron enzymes. An NADPH electron donor was suggested by early cyclase assays using biochemical fractions from Synechocystis, C. reinhardtii and plants ${ }^{13,15}$ but no activity was detected with just AcsF and NADPH (Fig. 2e; $4^{\text {th }}$ trace from top), suggesting that it is not directly involved in cyclase activity. Guided by the findings that a few diiron enzymes accept electrons from reduced Fd for activity ${ }^{27,28}$, we combined spinach Fd and FNR with NADPH to form a tricomponent Fd reduction system, which did support the cyclase activity of AcsF (Fig. 2e; top trace). The activity depends on the presence of NADPH, Fd, FNR and AcsF, as indicated by the control assays (Fig. $2 \mathrm{e} ; 2^{\text {nd }}, 3^{\text {rd }}, 4^{\text {th }}, 5^{\text {th }}$ traces from top). Next, we scaled up the assay and increased the MgPME concentration from 10 to $27 \mu \mathrm{M}$. The catalytic activity of AcsF is clearly demonstrated by the dramatic color change of the assay mixture indicating a likely complete conversion with only 30 min incubation (Fig. 2f).

\section{Steady-state kinetic behavior and porphyrin binding of AcsF}

The dramatic color change from red to green upon product formation allowed us to develop an absorption-based continuous assay to investigate the steady-state kinetic behavior of AcsF. We switched to a cyanobacterial source of Fd and FNR, by overexpressing the genes from Anabaena sp. PCC 7119 (hereafter Anabaena) in E. coli (see Extended Data Fig. 1 for electrophoretic analysis and absorbance spectra of purified Anabaena Fd and FNR). Test assays monitored the spectral changes and found a clear trend of decreased absorbance maxima of the substrate at 422, 552 and $592 \mathrm{~nm}$, accompanied by increasing absorbance maxima for the product at 446, 586 and $634 \mathrm{~nm}$ (Fig. 3a). The initial rate of product formation was calculated by quantifying DV PChlide $a$ absorbance at $634 \mathrm{~nm}$ using a reported extinction coefficient ${ }^{29,30}$. We observed clear linear dependency of the initial rate with respect to the AcsF concentration (Fig. 3b). As the electron mediator between NADPH and AcsF, Fd was found to be rate-limiting. The initial rate displayed a hyperbolic response to Fd with an apparent $K_{\mathrm{M}}$ determined to be $4.05 \pm 0.39 \mu \mathrm{M}$ in the presence of $0.17 \mu \mathrm{M}$ FNR or $2.41 \pm 0.26 \mu \mathrm{M}$ with $1.7 \mu \mathrm{M}$ FNR (Fig. 3c). In subsequent assays, Fd was used at a saturating concentration of $31 \mu \mathrm{M}$ along with $1.7 \mu \mathrm{M}$ FNR. The dependence of the initial rate on MgPME followed Michaelis-Menten kinetics with the characterising parameters $k_{\text {cat }}$ $=0.91 \pm 0.02 \mathrm{~min}^{-1}, K_{\mathrm{M}}=7.03 \pm 0.51 \mu \mathrm{M}, k_{\text {cat }} / K_{\mathrm{M}}=0.13 \pm 0.01 \mu \mathrm{M}^{-1} \mathrm{~min}^{-1}$ (Fig. 3d). A sigmoidal relationship was found between the initial rate and the NADPH concentration so the Hill equation was used to fit the kinetic data with $k_{\text {cat }}=1.06 \pm 0.01 \mathrm{~min}^{-1}, K_{0.5}=0.16$ $\pm 0.01 \mathrm{mM}, k_{\text {cat }} / K_{0.5}=6.64 \pm 0.21 \mathrm{mM}^{-1} \mathrm{~min}^{-1}$ and the Hill coefficient $n=2.1 \pm 0.1$ (Fig. 3e). 
AcsF is the only required subunit of the cyclase, so we quantified the binding affinity for the porphyrin substrate, MgPME, by measuring the tryptophan fluorescence quenching of AcsF upon binding of MgPME. With increasing concentrations of MgPME, the degree of quenching gradually intensified (Fig. 3f). The titration data fit a single-site binding model with modifications to include the inner filter effect of MgPME (refs. ${ }^{31,32}$ ), giving a dissociation constant $K_{\mathrm{d}}$ of $0.16 \pm 0.05 \mu \mathrm{M}$ for MgPME binding (Fig. 3g).

\section{Identification of the reaction intermediates of AcsF}

Apart from MgPME substrate and DV PChlide a product, some end-point cyclase assays contained two pigment species provisionally named X1 and X2 for the $\sim 21$ and $\sim 28 \mathrm{~min}$ HPLC peaks, respectively. Intriguingly, X1 had the same absorbance and fluorescence spectra as MgPME whereas the spectral characteristics of X2 were between those of MgPME and DV PChlide $a$ (Fig. 4; insets). The MgPME we used was highly pure and the other assay components were defined, which was supported by the absence of X1 and X2 in the control assay without AcsF (Fig. 4). Assays with two-fold escalating concentrations of AcsF were terminated after 30 min incubation. As shown in Fig. 4, peaks X1 and X2 became apparent with increasing concentrations of AcsF, reached a maximum level, then gradually disappeared, while the DV PChlide a product continuously accumulated. The dynamics of $\mathrm{X} 1$ and $\mathrm{X} 2$ during the progress of the reaction are consistent with possible roles as reaction intermediates. Based on their spectral features, we suspected X1 to be the hydroxy and X2 the keto reaction intermediates as previously proposed ${ }^{8,20}$.

To confirm the identity of $\mathrm{X} 1$ and $\mathrm{X} 2$, the assay with $0.92 \mu \mathrm{M}$ AcsF (4x AcsF), which accumulated the two pigment species the most (Fig. 4), was scaled up and the resulting pigment extract analysed by liquid chromatography-electrospray ionisation-tandem mass spectrometry (LC-ESI-MS/MS). Extracted ion chromatograms show that the putative MgPME, $13^{1}$-hydroxy-MgPME and $13^{1}$-keto-MgPME intermediates, and DV PChlide a were baseline resolved by reverse phase LC (Fig. 5a-d; left). Consistent with the identification of the extracted ion peak at $614.2 \mathrm{~m} / z$ as $13^{1}$-hydroxy-MgPME (Fig. 5b; left), its retention time at 24 min was significantly earlier than the other three tetrapyrroles, as expected by the greater hydrophilicity conferred by the presence of a hydroxyl group. The non-hydroxylated tetrapyrroles were all clustered in the later 29-33 min eluting region. Mass spectral criteria for validating our identification of MgPME, $13^{1}$-hydroxy-MgPME, $13^{1}$-keto-MgPME and DV PChlide $a$ are by comparison of the experimental and theoretical isotopomer $\mathrm{m} / \mathrm{z}$ values and relative intensities in the full-MS spectra (Supplementary Fig. 3a-d; top left). The monoisotopic ions showed accuracies of 0.49-0.84 ppm, and in three cases there was close agreement in isotopomer patterns. In the case of $13^{1}$-keto-MgPME (Supplementary Fig. 3c; top left) isotopomer pattern fidelity could not be assessed because the ${ }^{13} \mathrm{C}_{1}$ and ${ }^{13} \mathrm{C}_{2}$ ions were merged with coincident unidentified ions. MgPME, $13^{1}$ hydroxy-MgPME and $13^{1}$-keto-MgPME (Supplementary Fig. 3a-c; top left) all ionised in the electrospray source to form radical cations. In the case of DV PChlide $a$, the number of neutral molecules available for radical cation formation was lowered by a dominant population of protonated cations (Supplementary Fig. 3d; top left), presumably as a result of a change in electrochemical properties with cyclisation ${ }^{33}$. Product ion spectra generated by the higher-energy $\mathrm{C}$-trap dissociation (HCD) of the precursor ions indicate relatively simple 
neutral loss pathways for MgPME and DV PChlide a (Fig. 5a,d; centre and right) forming the predicted carbocation products in which the positive charge resides on the larger fragment. No side-chain signature product ions at 59 and $73 \mathrm{~m} / z$ were detected. Similarly, the putative $13^{1}$-hydroxy-MgPME and $13^{1}$-keto-MgPME molecular ions dissociated without generating low $\mathrm{m} / \mathrm{z}$ signature ions, however they did form a greater number (6-7) of high $\mathrm{m} /$ $z$ cation products (Fig. 5b,c; centre). The increased number of potential neutral loss pathways for $13^{1}$-hydroxy-MgPME and $13^{1}$-keto-MgPME is an expected consequence of the additional functional group on the $\mathrm{C} 13$ methylpropionyl side chain. We further validated these two intermediates (Fig. 5b,c; right) by mapping their product ion spectra to structures generated by these neutral loss pathways (Supplementary Fig. 3b,c; bottom).

\section{Coupling the cyclase to reduced Fd produced by Photosystem I}

We have shown the cyclase activity requires electrons supplied by reduced Fd (Fig. 2e), which in oxygenic phototrophs is generated by Photosystem I (PSI) using light energy and electrons from plastocyanin $(\mathrm{Pc})$. To test a direct link between the activities of cyclase and PSI, we conducted in vitro assays that coupled the cyclase assay with a reconstituted PSI electron transport system ${ }^{34}$, containing sodium L-ascorbate (Asc) as the reductant, 2,6dichlorophenolindophenol (DCPIP) as the electron mediator, spinach Fd, Pc and PSI. It is intriguing that even in the absence of PSI the two cyclase reaction intermediates, 131hydroxy-MgPME and 13 ${ }^{1}$-keto-MgPME, were detected (Extended Data Fig. 2 and Supplementary Fig. 4a). Systematic control assays showed that Asc alone was able to support a low level of cyclase activity, with conversion of $16 \%$ of the MgPME substrate to $13^{1}$-hydroxy-MgPME and $13^{1}$-keto-MgPME intermediates although with no formation of the final product (Supplementary Fig. 4b). However, the cyclase catalytic cycle was only completed, generating the final product DV PChlide $a$, in the presence of PSI and with light exposure (Extended Data Fig. 2). The production level of DV PChlide a clearly depended on the PSI level (Extended Data Fig. 2; 1x PSI versus 4x PSI) and was approximately proportional to the duration of light exposure (Extended Data Fig. 2; 15 min light versus 30 min light). These results clearly show the potential for the cyclase step in chlorophyll biosynthesis to be coupled to production of reduced Fd and turnover of PSI, a point that that will be covered in the Discussion.

\section{Discussion}

Over 70 years have elapsed since Granick's proposed reaction sequence for the formation of the isocyclic ring of chlorophyll ${ }^{8}$. The inability to dissect this sequence represented a large gap in our knowledge of chlorophyll biosynthesis, given that formation of ring E starts to establish the eventual absorption lineshape of chlorophyll. The presence of ring E, a prerequisite for all chlorins and bacteriochlorins, extends the $\pi$ system along the $\mathrm{Q}_{\mathrm{y}}$ axis, red-shifting and enhancing the $\mathrm{Q}_{\mathrm{y}}$ absorption band. The availability of a biochemically pure $\mathrm{O}_{2}$-dependent cyclase represented the only prospect resolving the reaction intermediates, and recent genetic approaches that resolved the enigmatic subunit composition of the $\mathrm{O}_{2^{-}}$ dependent cyclase ${ }^{4,21}$ paved the way for recombinantly producing the enzyme for in vitro analysis. In the present study, we overexpressed a single-subunit cyclase, the $R v i$. gelatinosus AcsF, in E. coli and purified the enzyme to homogeneity. The pale brown colour 
and iron content of the purified AcsF protein, combined with the $\sim 340 \mathrm{~nm}$ charge transfer band as well as the distinctive absorbance bands when in complex with azide, provide experimental evidence that AcsF is indeed a diiron protein. Sequence alignments of some AcsF proteins and their putative diiron ligands are shown in Extended Data Fig. 3a, and Extended Data Fig. 3b,c show how the diiron ligands of AcsF might be coordinated, based on motifs shared between AcsF proteins and the soluble methane monooxygenase hydroxylase subunit from Methylococcus capsulatus.

AcsF is a monooxygenase so $\mathrm{O}_{2}$ activation is part of its catalytic cycle, which requires a source of electrons. Our reconstitution tests showed that NADPH can be the ultimate electron source but not the direct electron donor as an oxidoreductase, FNR, and an electron mediator, $\mathrm{Fd}$, were also required to form the electron transfer chain that supports cyclase activity (Fig. 2e). We were able to reconstitute the cyclase activity using either the spinach FNR and Fd (Fig. 2) or recombinantly produced Anabaena counterparts (Fig. 3) which, taken together with the in vivo heterologous activity of AcsF shown in E. coli (ref. ${ }^{21}$ ), indicate that the AcsF reaction likely shares a generic electron transfer chain with other metabolic processes. In its native host, Rvi. gelatinosus, AcsF relies on the reduced Fd generated by the Rnf system ${ }^{35}$, which utilises the protonmotive force to derive electrons from NADH to Fd, and/or a flavin-based electron bifurcation system ${ }^{36}$, which oxidises $\mathrm{NADH}$ to produce reduced $\mathrm{Fd}$ and ubiquinol simultaneously. The cyclase from oxygenic phototrophs is expected to also use reduced Fd as the electron source, given that cyanobacterial, green algal and plant cyclases are active in $E$. coli (refs. ${ }^{21,37}$ ), and cyanobacterial and plant cyclases are functional in Rvi. gelatinosus (refs. ${ }^{4,37}$ ). Intriguingly, a recent report has already connected the cyclase activity with the plastidal FNR in plants ${ }^{38}$.

Furthermore, while our paper was being reviewed, a report emerged showing that the in vitro activity of barley cyclase is Fd-dependent ${ }^{39}$. It is conceivable that the requirement for additional electron transfer partners eluded early cyclase characterisations that used complex cellular fractions. The finding of the Fd involvement is significant as it points to a possible connection between the cyclase and the photosynthetic electron transport chain, linking chlorophyll biosynthesis to photosynthetic activity and/or the redox state of thylakoid membranes ${ }^{40}$. Despite this possible direct link to PSI, the NADPH/FNR/Fd was the most effective source of electrons for assaying the cyclase in vitro (Fig. 4) which, if replicated in vivo, would allow the 'dark' synthesis of DV PChlide a. This route for Fd reduction is shown in Fig. 6, in addition to a more direct, PSI-coupled source of electrons. The ultimate source of reduced FNR and Fd is PSI, and the $\mathrm{O}_{2}$-dependent cyclase adds a candidate to the list of metabolic functions supported by PSI (ref. ${ }^{41}$ ). As proof of principle, we devised an assay that couples the catalytic cycle of the cyclase with provision of reduced Fd by PSI (Extended Data Fig. 2). Fig. 6 depicts this dependence of chlorophyll biosynthesis on PSI turnover, and it shows that the light-dependent stage in chlorophyll biosynthesis, catalysed by light-dependent PChlide oxidoreductase (LPOR), is preceded by a cyclase step that, albeit indirectly, is also light-dependent.

The AcsF cyclase displays classic Michaelis-Menten kinetic behaviour regarding the porphyrin substrate MgPME. The Michaelis constant, $K_{\mathrm{M}}$, was determined to be $7.0 \mu \mathrm{M}$ (Fig. 3d), typical of chlorophyll biosynthetic enzymes, such as LPOR and magnesium chelatase from Synechocystis with $K_{\mathrm{M}}$ values of $8.6 \mu \mathrm{M}$ for PChlide (ref. ${ }^{42}$ ) and $3.2 \mu \mathrm{M}$ 
for deuteroporphyrin IX (ref. ${ }^{43}$ ), respectively. The $K_{\mathrm{d}}$ for MgPME binding to AcsF is 0.16 $\mu \mathrm{M}$ (Fig. 3f,g), somewhat lower than for magnesium chelatase $\left(K_{\mathrm{d}}\right.$ of $1.2 \mu \mathrm{M}$ for deuteroporphyrin IX $)^{31}$, and the methyltransferase $\left(K_{\mathrm{d}}\right.$ of $2.4 \mu \mathrm{M}$ for Mg-deuteroporphyrin IX $)^{44}$. The $K_{\mathrm{M}}$ for MgPME greatly exceeds $K_{\mathrm{d}}$, due to the complexity of the reaction undertaken by AcsF, and its reliance on both FNR and Fd during the reaction cycle. This $K$ $M$ value is also likely influenced by $\beta$-DDM detergent molecules associated with AcsF and with the hydrophobic MgPME substrate, with consequent effects on product release. Thus, the low turnover number, $k_{\text {cat }}$, of $0.9 \mathrm{~min}^{-1}$ with respect to MgPME (Fig. 3d), reflects the complexity of the catalytic sequence that consists of multiple sequential reactions, each of which requires two electrons supplied by a coupled redox reaction. The value of $k_{\text {cat }}$ is comparable with the $0.8 \mathrm{~min}^{-1}$ measured for magnesium chelatase ${ }^{43}$, both of which are much slower than the $57 \mathrm{~s}^{-1}$ obtained for the methyltransferase $\mathrm{s}^{44,45}$, which precedes the cyclase in the chlorophyll biosynthesis pathway. As for NADPH, the kinetic data were best described by the Hill equation and showed positive cooperativity $(n=2.1)$ (Fig. 3e), which arises from the multiple sequential NADPH-dependent reactions required to form the final product.

The availability of an in vitro cyclase assay enabled us to isolate and identify the progression of chemical species en route to the DV PChlide a product. Amounts of purified enzyme and porphyrin substrate could be varied to adjust the levels of cyclase reaction intermediates and final product, and the MS identification method minimised the possible interference from undefined protein components, pigment impurities and artefacts. We conclusively identified $13^{1}$-hydroxy-MgPME and $13^{1}$-keto-MgPME as reaction intermediates on the basis of the $\mathrm{m} /$ $z$ of their radical cations and the specific transition of the precursor ion to product ions (Fig. 5 and Supplementary Fig. 3). As the reaction proceeded these two pigment species showed the transition from initial accumulation to subsequent dissipation (Fig. 4), characteristic of reaction intermediates. The hydrophobicity and spectral features of $13^{1}$-hydroxy-MgPME are consistent with previous studies involving the cucumber and $C$. reinhardtii cyclase systems (Fig. 4; peak X1) ${ }^{14,46} .13^{1}$-keto-MgPME was demonstrated to be a cyclase substrate with chemically synthesised pigments ${ }^{14}$ but was not directly detected in any cyclase system until now. The spectroscopic properties of $13^{1}$-keto-MgPME (Fig. 4; peak X2) match those of the synthetic version ${ }^{14}$, and position the pigment between MgPME and DV PChlide a, reminiscent of the 'longer wavelength metalloporphyrins' detected in cucumber cotyledons ${ }^{47}$. We did not detect any pigment species suggestive of a $13^{1}-13^{2}$ acrylate derivative of MgPME in the assay by HPLC or MS analysis, consistent with the report that the synthetic acrylate derivative is not a cyclase substrate ${ }^{20}$. The reaction sequence, signposted by the reaction intermediates identified by MS, is depicted in Fig. 6a. We note that although there is no final product, DV PChlide a, Asc can support a limited (16\%) conversion of MgPME substrate, forming low levels of the $13^{1}$-hydroxy and $13^{1}$-keto intermediates (Supplementary Fig. 4b), possibly indicating different redox requirement for the three steps of the catalytic cycle. Although the in vitro assay with FNR/Fd can convert all of the MgPME to DV PChlide a, further work is required to investigate the electron donors in vivo, and the mechanism by which reduced Fd serves as the direct electron donor to the diiron centre of AcsF. Mechanistic details of the activation of molecular oxygen 
within the AcsF cyclase, a diiron enzyme, require the application of spectroscopic and structural techniques.

In summary, our work removes the last remaining hurdle in the study of the $\mathrm{O}_{2}$-dependent cyclase by showing reduced Fd serves as the direct electron donor to the diiron centre of AcsF. Our approaches for expression, purification and reconstitution of cyclase are transferrable to the study of the other two classes of $\mathrm{O}_{2}$-dependent cyclase, which require auxiliary Ycf54 or BciE subunits ${ }^{4}$. Our unambiguous identification of the two reaction intermediates provides an insight into the catalytic mechanism, which should be further investigated by mutagenesis and structural studies in order to pinpoint the key residues involved in formation of the isocyclic ring. In addition, a detailed analysis of the interaction between Fd and AcsF should be performed in the future to establish the Fd binding site that promotes electron transfer during the catalytic cycle.

\section{Materials and Methods}

\section{Production and purification of AcsF, Fd and FNR}

The acsF gene (RGE_33550) was amplified from the genomic DNA of Rvi. gelatinosus using the forward primer 5'-GAGTCTCATATGCTCGCGACCCCGACGATCG-3' and the reverse primer 5'-GAGTCTGGATCCTCACCATGCCGGGGCCATG-3', and cloned into the NdeI/BamHI sites of the pET14b vector, resulting in the pET14b-AcsF plasmid. Gene fragments encoding the Anabaena Fd (P0A3C8) and FNR (P21890; lacking the N-terminal 136 aa) were synthesised (Integrated DNA Technologies) with codon optimisation for expression in E. coli, and cloned into the NcoI/HindIII sites of the pET28a vector to get the pET28a-Fd and pET28a-FNR plasmids, respectively. The nucleotide sequences of synthesised genes are listed in Supplementary Table 1. The E. coli C43(DE3), BL21(DE3) and BL21(DE3) $\Delta i s c R$ (ref. ${ }^{48}$ ) strains were used for overexpression of AcsF, FNR and Fd, respectively. E. coli strains were grown in TB medium $\left(12 \mathrm{~g} \mathrm{~L}^{-1}\right.$ tryptone, $24 \mathrm{~g} \mathrm{~L}^{-1}$ yeast extract, $10 \mathrm{~g} \mathrm{~L}^{-1}$ glycerol, $2.31 \mathrm{~g} \mathrm{~L}^{-1} \mathrm{KH}_{2} \mathrm{PO}_{4}, 12.54 \mathrm{~g} \mathrm{~L}^{-1} \mathrm{~K}_{2} \mathrm{HPO}_{4}$ ) for AcsF and $\mathrm{Fd}$, and in LB medium for FNR, with $100 \mu \mathrm{g} \mathrm{mL}-1$ ampicillin or $30 \mu \mathrm{gL}^{-1}$ kanamycin where required. Cultures were grown at $37^{\circ} \mathrm{C}$ with shaking at $220 \mathrm{rpm}$ then shifted to either $30^{\circ} \mathrm{C}$ for AcsF and FNR, or $28^{\circ} \mathrm{C}$ for $\mathrm{Fd}$, with shaking at $175 \mathrm{rpm}$ for induction. For C43(DE3)/ pET14b-AcsF, $1 / 50$ of starter culture was inoculated and the culture grown for $5 \mathrm{~h}$ before 0.4 $\mathrm{mM}$ isopropyl- $\beta$-D-thiogalactopyranoside (IPTG) and $0.1 \mathrm{~g} \mathrm{~L}^{-1}$ ferric ammonium citrate were added. After $24 \mathrm{~h}$ induction, cells were harvested and resuspended in buffer A [ $25 \mathrm{mM}$ MOPS-NaOH, pH 7.5, $10 \mathrm{mM} \mathrm{MgCl}_{2}, 500 \mathrm{mM} \mathrm{NaCl}, 10 \%$ (vol/vol) glycerol, $20 \mathrm{mM}$ imidazole]. For BL21(DE3)/pET28a-FNR, a 1/100 inoculum was used and the culture grown to an $\mathrm{OD}_{600}$ of $0.6-0.8$ before induction with $0.25 \mathrm{mM}$ IPTG. Cells were harvested after $20 \mathrm{~h}$ induction and resuspended in $20 \mathrm{mM}$ Tris- $\mathrm{HCl} \mathrm{pH} \mathrm{9.0.} \mathrm{A} \mathrm{1/100} \mathrm{inoculum} \mathrm{was}$ used for BL21(DE3) $\Delta i s c R /$ pET28a-Fd, and the culture grown to an $\mathrm{OD}_{600}$ of $0.6-0.8$ before supplementation with $0.25 \mathrm{mM}$ IPTG and $0.1 \mathrm{~g} \mathrm{~L}^{-1}$ ferric ammonium citrate. After 24 $\mathrm{h}$ induction, cells were harvested and resuspended in $20 \mathrm{mM}$ Tris- $\mathrm{HCl} \mathrm{pH} \mathrm{7.4.} \mathrm{All} \mathrm{cell}$ suspensions were flash-frozen in liquid $\mathrm{N}_{2}$ and stored at $-20{ }^{\circ} \mathrm{C}$.

For purification of AcsF, cells were defrosted, and supplemented with DNase I, lysozyme and proteinase inhibitor cocktail (Sigma-Aldrich) before incubation at room temperature 
with shaking for $20 \mathrm{~min}$. Cells were disrupted by one passage through a French pressure cell at $18,000 \mathrm{psi}$ and 8 cycles of $30 \mathrm{~s}$ sonication, followed by centrifugation at $43,399 \times g$ at 10 ${ }^{\circ} \mathrm{C}$ for $30 \mathrm{~min}$. The resulting pellet was resuspended in buffer $\mathrm{A}$. Then $\beta$-DDM was added at a concentration of $1 \%(\mathrm{wt} / \mathrm{vol})$ and solubilisation performed at $4{ }^{\circ} \mathrm{C}$ on a tube roller for $1 \mathrm{~h}$. Insoluble material was removed by centrifugation at $43,399 \times g$ at $10{ }^{\circ} \mathrm{C}$ for $30 \mathrm{~min}$. The soluble fraction was diluted $2 \times$ with buffer $\mathrm{A}$ and applied to $\mathrm{Ni}^{2+}$-loaded Chelating Sepharose Fast Flow resin (GE Healthcare), washed with buffer B ( $25 \mathrm{mM}$ MOPS-NaOH, $\mathrm{pH} 7.5,10 \mathrm{mM} \mathrm{MgCl} 2,500 \mathrm{mM} \mathrm{NaCl}, 10 \%$ glycerol, $100 \mathrm{mM}$ imidazole, $0.04 \% \beta$-DDM) and protein eluted with buffer $\mathrm{C}$ ( $25 \mathrm{mM}$ MOPS-NaOH, pH 7.5, $10 \mathrm{mM} \mathrm{MgCl}_{2}, 100 \mathrm{mM}$ $\mathrm{NaCl}, 10 \%$ glycerol, $400 \mathrm{mM}$ imidazole, $0.04 \% \beta$-DDM). Protein-containing fractions were pooled and exchanged into buffer $\mathrm{D}\left(50 \mathrm{mM}\right.$ MES-NaOH, pH 6.0, $10 \mathrm{mM} \mathrm{MgCl}_{2}, 500 \mathrm{mM}$ $\mathrm{NaCl}, 10 \%$ glycerol, $0.04 \% \beta$-DDM) using a PD-10 column (GE Healthcare). After adding $2 \mathrm{mM}$ ferrous ammonium sulphate, the protein solution was incubated at $4{ }^{\circ} \mathrm{C}$ on a tube roller for $1 \mathrm{~h}$, clarified by centrifugation at $21,380 \times g$ at $4{ }^{\circ} \mathrm{C}$ for $10 \mathrm{~min}$, and loaded onto a HiLoad 16/600 Superdex 200 prep grade gel filtration column (GE Healthcare) equilibrated with buffer D and eluted at $0.4 \mathrm{~mL} \mathrm{~min}^{-1}$ using an ÄKTAprime plus instrument monitored by PrimeView 5.0 (hereafter for gel filtration and ion exchange chromatography). Soluble protein standards from the Gel Filtration Markers Kit for 29-700 kDa (Sigma-Aldrich) and membrane protein standards kindly provided by Dr David Swainsbury, University of Sheffield, were analysed using the same gel filtration method. Calibration curves were generated using SigmaPlot 14.0 and used to estimate the molecular mass of AcsF whose concentration was determined by absorbance at $280 \mathrm{~nm}$ using an extinction coefficient of $59,820 \mathrm{M}^{-1} \mathrm{~cm}^{-1}$, calculated by the ProtParam tool in the ExPASy portal ${ }^{49}$.

For purification of FNR, cell breakage was conducted as per the purification of AcsF but the passage through a French pressure cell was omitted. The cell lysate was clarified by centrifugation at $43,399 \times g$ at $10{ }^{\circ} \mathrm{C}$ for $30 \mathrm{~min}$, passed through a $0.45 \mu \mathrm{m}$ filter, and applied to a HiTrap Q HP anion exchange column (GE Healthcare). The column was washed with $20 \mathrm{mM}$ Tris- $\mathrm{HCl} \mathrm{pH} 9.0$ and proteins were eluted with a linear gradient of $0-0.25 \mathrm{M} \mathrm{NaCl}$ in $20 \mathrm{mM}$ Tris-HCl pH 9.0 over $200 \mathrm{~mL}$. Fractions containing FNR were pooled and concentrated before loading onto a HiLoad 16/600 Superdex 200 prep grade gel filtration column (GE Healthcare) equilibrated with buffer E (20 mM HEPES-NaOH, pH 7.5, 100 $\mathrm{mM} \mathrm{NaCl}$ ) and eluted at $0.5 \mathrm{~mL} \mathrm{~min}^{-1}$. For purification of $\mathrm{Fd}$, cell breakage was conducted as for the purification of AcsF. The cell lysate was clarified and subjected to anion exchange chromatography as for the purification of FNR except that a linear gradient of $0-0.5 \mathrm{M} \mathrm{NaCl}$ in $20 \mathrm{mM}$ Tris- $\mathrm{HCl}$ pH 7.4 over $200 \mathrm{~mL}$ was used for elution. Fractions containing Fd were pooled and subjected to ammonium sulphate precipitation three times at $0{ }^{\circ} \mathrm{C}$ to remove contaminants. Solid ammonium sulphate was directly added to the protein solution until cloudy and protein contaminants were removed by centrifugation. Fd was recovered from the clarified solution by performing ammonium sulphate precipitation twice with $100 \%$ saturated solution at $0{ }^{\circ} \mathrm{C}$, reconstituted with $\sim 5 \mathrm{ml} 20 \mathrm{mM}$ Tris- $\mathrm{HCl} \mathrm{pH} 7.4$ and purified by gel filtration as for FNR. Fd-containing fractions were pooled and subjected to ammonium sulphate precipitation twice with $100 \%$ saturated solution at $0{ }^{\circ} \mathrm{C}$ to remove potential nucleic acid contamination. The recovered Fd was finally dissolved in buffer E. Concentrations of cofactor-containing FNR and Fd were determined by absorbance using reported extinction 
coefficients of 9,400 $\mathrm{M}^{-1} \mathrm{~cm}^{-1}$ at $458 \mathrm{~nm}$ for FNR, and 7,200 M $\mathrm{M}^{-1} \mathrm{~cm}^{-1}$ at $423 \mathrm{~nm}$ for Fd (ref. ${ }^{50}$ ).

\section{Iron quantification}

The iron content of the purified AcsF protein was determined using an iron assay kit (Sigma-Aldrich) according to the manufacturer's instructions. Assays were conducted in triplicate and data was analysed using SigmaPlot 14.0.

\section{Absorbance spectroscopy}

Spectra were recorded on either a Cary 60 UV-vis spectrophotometer (Cary WinUV Scan Application 5.1.0.1016) or an Omega FluoStar microplate reader (BMG LABTECH, Reader Control software 5.50 R4) equipped with an LVis plate. To record the AcsF-sodium azide spectrum, $16 \mu \mathrm{M}$ AcsF was mixed with an equal volume of $4 \mathrm{M}$ sodium azide stock solution prepared in buffer $\mathrm{D}$ and the mixture was incubated at room temperature for $30 \mathrm{~min}$. The absorbance spectrum was recorded using $2 \mathrm{M}$ sodium azide in buffer $\mathrm{D}$ as blank.

\section{Purification of MgPME}

MgPME was extracted from a Rvi. gelatinosus $\triangle b c h E \Delta a c s F$ mutant as described previously ${ }^{21}$. The resulting MgPME solution was vacuum dried, reconstituted in a minimal volume of $0.2 \%(\mathrm{wt} / \mathrm{vol})$ ammonia in methanol and purified on an Agilent $1200 \mathrm{HPLC}$ system (ChemStation for LC 3D systems B.04.02) using a Fortis $\mathrm{C}_{18}$ reverse-phase column (particle size $5 \mu \mathrm{m} ; 150 \mathrm{~mm} \times 10 \mathrm{~mm}$ ). Pigments were eluted at $40{ }^{\circ} \mathrm{C}$ at $2.5 \mathrm{~mL} \mathrm{~min}{ }^{-1}$ with a linear gradient from $35 \%$ (vol/vol) solvent A [350 mM ammonium acetate, $30 \%$ (vol/vol) methanol] to $75 \%$ (vol/vol) solvent B (methanol) over $35 \mathrm{~min}$ and monitored by absorbance at $416 \mathrm{~nm}$. Fractions containing $\mathrm{MgPME}$ were collected, mixed with $0.5 \mathrm{vol} \mathrm{QH}_{2} \mathrm{O}$, and loaded onto a Discovery DSC-18 SPE tube column (Sigma-Aldrich) for solid phase extraction. The column was washed with $\mathrm{QH}_{2} \mathrm{O}$ to remove ammonium acetate. MgPME was eluted with methanol, vacuum dried and stored at $-20^{\circ} \mathrm{C}$. MgPME concentration was estimated by absorbance at $589 \mathrm{~nm}$ using an extinction coefficient of $18,000 \mathrm{M}^{-1} \mathrm{~cm}^{-1}$ in methanol ${ }^{11}$.

\section{Differential scanning calorimetry}

The purified AcsF was centrifuged at $16,000 \times g$ at $4{ }^{\circ} \mathrm{C}$ for 10 min to remove any possible aggregates and diluted to $1 \mathrm{mg} \mathrm{mL}^{-1}$ in buffer D. $300 \mu \mathrm{L}$ of the diluted AcsF solution was loaded into a NanoDSC (TA Instruments) and subjected to a heat ramp of $1{ }^{\circ} \mathrm{C} \mathrm{min}^{-1}$. After removing the start-up hook, the data was converted to molar heat capacity using NanoAnalyze 3.11.0 and exported to Igor Pro 8.04 for processing by a cubic spline baseline fit followed by a Gaussian fit, whose modal value was reported as the melting point.

\section{End-point HPLC-based cyclase assays}

Spinach Fd, FNR, bovine catalase and NADPH were from Sigma-Aldrich. Assays were

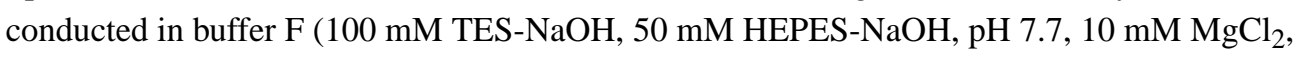
$1 \mathrm{M}$ glycerol, $0.04 \% \beta$-DDM) with $3.7 \mu \mathrm{M}$ AcsF, $0.2 \mathrm{mg} \mathrm{mL}^{-1}$ spinach Fd, 0.4 units mL $\mathrm{m}^{-1}$ spinach FNR, $2 \mathrm{mM}$ NADPH, $10 \mu \mathrm{M}$ MgPME (added from $200 \mu \mathrm{M}$ MgPME stock solution 
prepared in $0.2 \%$ ammonia in methanol) and $0.29 \mathrm{mg} \mathrm{mL}^{-1}$ catalase. To test the cofactor requirement for cyclase activity, AcsF, Fd, FNR and NADPH were omitted where indicated. For reaction intermediate determination, $20 \mu \mathrm{M}$ MgPME was used and AcsF was added at incrementally doubled concentrations from 0.23 to $3.68 \mu \mathrm{M}$. Assays were performed in 50 $\mu \mathrm{L}$ volume in $1.5 \mathrm{~mL}$ Eppendorf tubes and initiated with the addition of AcsF, followed by incubation at $30{ }^{\circ} \mathrm{C}$ in the dark with shaking at $175 \mathrm{rpm}$ for $30 \mathrm{~min}$. Then $200 \mu \mathrm{L}$ of $0.2 \%$ ammonia in methanol was added to stop the assay and $35 \mu \mathrm{L}$ of the clarified pigment extract was analysed by HPLC as described previously ${ }^{37}$. The elution of pigment species was monitored by absorbance at 416 and $440 \mathrm{~nm}$ and fluorescence emission at 595 and $640 \mathrm{~nm}$ (excitation at $440 \mathrm{~nm}$ ).

\section{Coupled PSI-cyclase assays}

Asc and DCPIP were from Sigma-Aldrich. Purified spinach PSI and Pc were kindly provided by Dr Guy Mayneord, University of Sheffield. Assays were conducted in buffer F with $2 \mu \mathrm{M}$ AcsF, $0.04 \mathrm{mg} \mathrm{mL}^{-1}$ spinach Fd, $14 \mu \mathrm{M}$ MgPME, spinach PSI containing 6 or 22.4 $\mu \mathrm{M}$ Chl a, $20 \mu \mathrm{M}$ spinach Pc, $2 \mathrm{mM}$ Asc, $60 \mu \mathrm{M}$ DCPIP and $0.29 \mathrm{mg} \mathrm{mL}^{-1}$ catalase. AcsF, Fd, PSI, Pc, Asc and DCPIP were omitted where indicated to test the cofactor dependency of cyclase activity. Assays were performed in $50 \mu \mathrm{L}$ volume in $1.5 \mathrm{~mL}$ Eppendorf tubes and incubated at $30^{\circ} \mathrm{C}$ either in the dark for $30 \mathrm{~min}$, or under illumination from two red LED bicycle taillights (50 lumens, WQJifv) for 15 or $30 \mathrm{~min}$. Assays were stopped by adding 4 vol $0.2 \%$ ammonia in methanol and $20 \mu \mathrm{L}$ of the clarified pigment extract was analysed by HPLC as described previously ${ }^{21}$. Pigment elution was monitored by fluorescence emission at $640 \mathrm{~nm}$ (excitation at $440 \mathrm{~nm}$ ).

\section{Continuous absorbance-based cyclase assays}

Assays were performed in buffer F with AcsF, Anabaena Fd, Anabaena FNR, NADPH, and MgPME at concentrations specified in the figure legends, and with catalase at $0.29 \mathrm{mg} \mathrm{mL}^{-1}$. Assays were conducted at $30^{\circ} \mathrm{C}$ in $100 \mu \mathrm{L}$ volume in Greiner $\mu$ clear F-bottom medium binding 96-well black microplates. Assays were initiated by adding AcsF and the reaction progress was monitored using an Omega microplate reader (BMG LABTECH, Reader Control software $5.50 \mathrm{R} 4$ ) in absorbance mode for $30 \mathrm{~min}$. Spectra from 400 to $750 \mathrm{~nm}$ were recorded for each well every 30 to $60 \mathrm{~s}$ (depending on the number of assays). Initial rates ( $V$ i) were calculated using the software supplied by the manufacturer (MARS 3.32 R5). Kinetic parameters were determined by fitting equation 1 to the data with nonlinear regression using Igor Pro 8.04. Errors were determined from least squares analysis of the fits. DV PChlide a concentration was estimated by absorbance at $634 \mathrm{~nm}$ using an extinction coefficient of $19,796 \mathrm{M}^{-1} \mathrm{~cm}^{-1}$ (refs. ${ }^{29,30}$ ).

$$
\begin{gathered}
v_{\mathrm{i}}=\frac{k_{\mathrm{cat}}[\mathrm{E}][\mathrm{S}]}{K_{\mathrm{M}}+[\mathrm{S}]} \\
v_{\mathrm{i}}=\frac{k_{\mathrm{cat}}[\mathrm{E}][\mathrm{S}]^{n}}{\left(K_{0.5)^{n}+[\mathrm{S}]^{n}}\right.}
\end{gathered}
$$

(equation 1) 
Where $v_{\mathrm{i}}$ is the initial reaction rate, $k_{\text {cat }}$ is the turnover number, $K_{\mathrm{M}}$ is the Michaelis constant, $n$ is the Hill coefficient, and $K_{0.5}$ is the substrate concentration that gives halfmaximal reaction rate in the Hill equation.

\section{Tryptophan fluorescence quenching binding assays}

Assays were conducted by mixing $0.2 \mu \mathrm{M}$ AcsF in buffer D with an equal volume of MgPME solution prepared in buffer G $[0.02 \%$ (wt/vol) ammonia, $10 \%$ (vol $/ \mathrm{vol}$ ) methanol, $90 \%$ (vol/vol) buffer D] at concentrations incrementally doubled from $40 \mathrm{nM}$ to $80 \mu \mathrm{M}$. The mixture was incubated at $30^{\circ} \mathrm{C}$ for $2 \mathrm{~min}$ and then fluorescence spectra between 300 and $400 \mathrm{~nm}$ (10 nm bandpass) were recorded on a FluroMax 3 fluorimeter (HORIBA Jobin Yvon) (FluorEssence Package 3.9) at $30{ }^{\circ} \mathrm{C}$ with excitation at $280 \mathrm{~nm}$ ( $5 \mathrm{~nm}$ bandpass). A modified single-site binding equation (equation 3 ), which takes into account the inner filter effect of light absorbance by MgPME (refs. ${ }^{31,32}$ ), was fitted to the obtained titration data with nonlinear regression using Igor Pro 8.04.

$$
F_{\mathrm{obs}}=F_{0}+F_{\max } \frac{[\mathrm{L}]_{\mathrm{T}}+[\mathrm{E}]_{\mathrm{T}}+K_{\mathrm{d}}-\sqrt{\left([\mathrm{L}]_{\mathrm{T}}+[\mathrm{E}]_{\mathrm{T}}+K_{\mathrm{d}}\right)^{2}-4[L]_{\mathrm{T}}[\mathrm{E}]_{\mathrm{T}}}}{2[\mathrm{E}]_{\mathrm{T}}}+M[\mathrm{~L}]_{\mathrm{T}} \quad \text { (equation 3) }
$$

Where $F_{\mathrm{obs}}$ is the observed fluorescence, $F_{0}$ is the initial fluorescence, $F_{\max }$ is the maximum amplitude of fluorescence quenching, $[\mathrm{L}]_{\mathrm{T}}$ is the total ligand concentration, $[\mathrm{E}]_{\mathrm{T}}$ is the total enzyme concentration (fixed at $0.1 \mu \mathrm{M}$ during the fitting procedure), $K_{\mathrm{d}}$ is the apparent dissociation constant, and $M$ is the inner filter contribution of ligand.

\section{Pigment analysis by LC-ESI-MS/MS}

Scaled-up in vitro assays were conducted as per the end-point HPLC-based cyclase assay with $0.92 \mu \mathrm{M}$ AcsF. The resulting pigment extract was mixed with 2 vol $\mathrm{QH}_{2} \mathrm{O}$ and subjected to solid phase extraction as per MgPME purification. Pigments were eluted with methanol, followed by vacuum drying and reconstitution in $50 \mu \mathrm{L} \mathrm{70 \%} \mathrm{(vol/vol)} \mathrm{methanol}$ (LC grade), of which $5 \mu \mathrm{L}$ was analysed by capillary-flow liquid chromatography (Dionex RSLCnano system, Thermo Scientific) coupled on-line to a Q Exactive HF quadrupoleOrbitrap mass spectrometer (Thermo Scientific). Analytes were separated on a Luna $\mathrm{C}_{18}$ reverse-phase column (particle size $5 \mu \mathrm{m}$; pore size $100 \AA$; $250 \mathrm{~mm} \times 1 \mathrm{~mm}$; Phenomenex) operating at $50 \mu \mathrm{L} \mathrm{min}-1$ and $40{ }^{\circ} \mathrm{C}$ with a linear gradient from $35 \%$ (vol/vol) solvent $A$ to $75 \%$ (vol/vol) solvent B over $35 \mathrm{~min}$. The mass spectrometer was controlled by Thermo Xcalibur 4.0.27.42 and fitted with a HESI source operating with the following parameters: spray voltage $3,500 \mathrm{~V}$ positive, capillary temperature $320^{\circ} \mathrm{C}$, sheath gas 35 units. For fullscan profile MS acquisition, the following parameters were used: range $500-700 \mathrm{~m} / \mathrm{z}$, resolution 120,000, automatic gain control target 1e6 and maximum fill time $200 \mathrm{~ms}$. Product ion scans were by centroid parallel reaction monitoring with selection of ions at 598.24, 614.24, 612.22 and $611.21 \mathrm{~m} / z$ for MgPME, $13^{1}$-hydroxy-MgPME, $13^{1}$-ketoMgPME and DV PChlide a, respectively, and at an isolation width of $1.2 \mathrm{~m} / z$. Other parameters were set to: resolution 30,000, automatic gain control target 2e5, maximum fill time $100 \mathrm{~ms}$ and stepped collision energy 30/35/40 eV. Mass spectra were extracted from the output data files and compared with theoretical relative isotopomer ion intensity values using Xcalibur 4.0.27.42. Mapping precursor and product ion masses to their structures was 
carried out with the aid of ACD/ChemSketch 2019.1.3. These structures were exported to Xara Xtreme 5.1.1.9166 to produce figures.

\section{Extended Data}

a

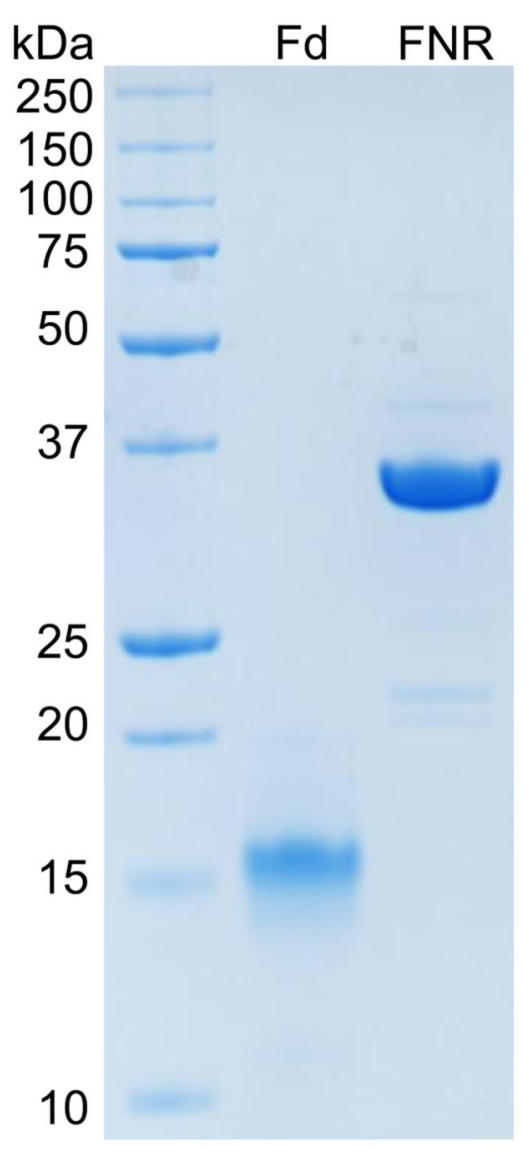

b

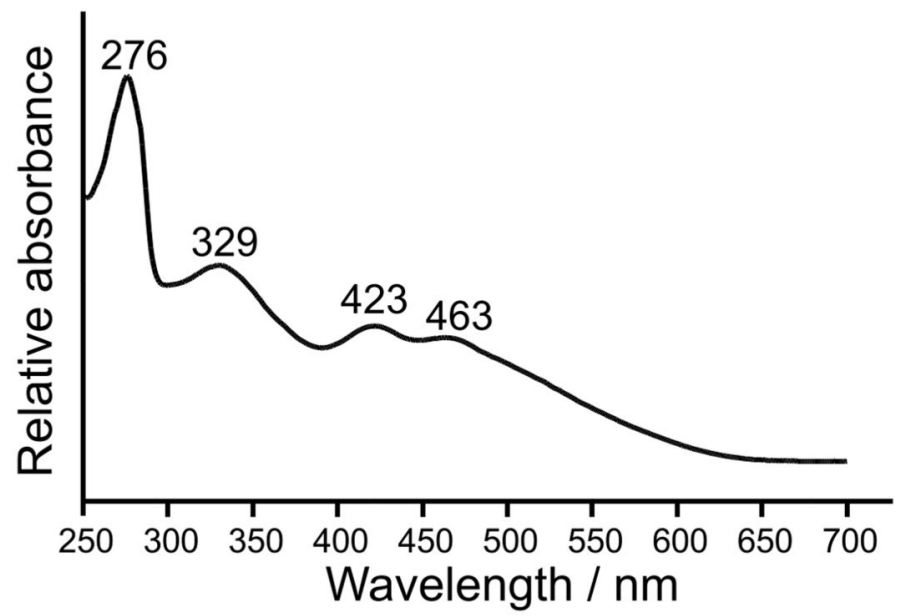

C

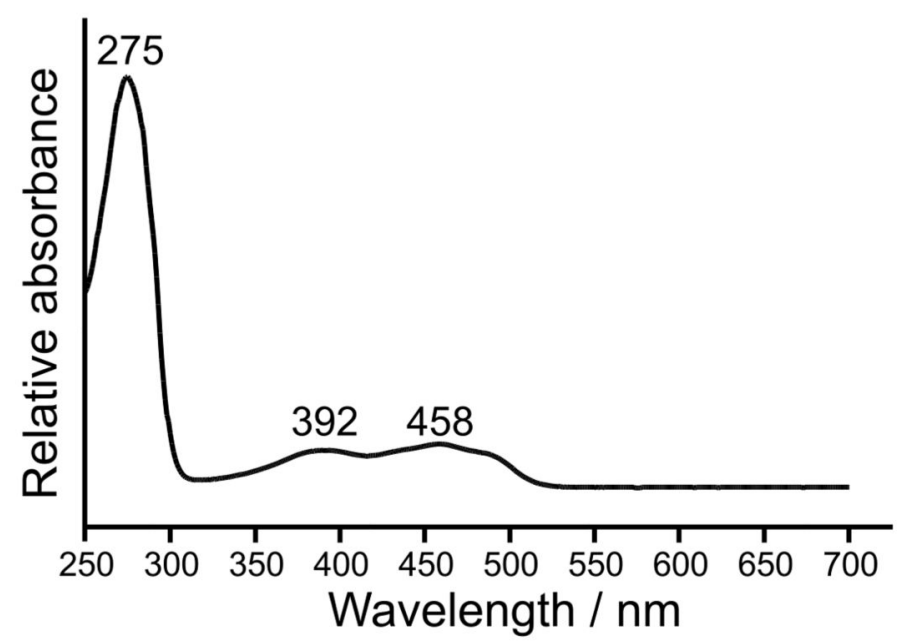

Extended Data Fig. 1. Analysis of purified Anabaena Fd and FNR.

a, SDS-PAGE analysis of purified Anabaena Fd and FNR. b, Absorbance spectrum of purified Anabaena Fd. c, Absorbance spectrum of purified Anabaena FNR. 


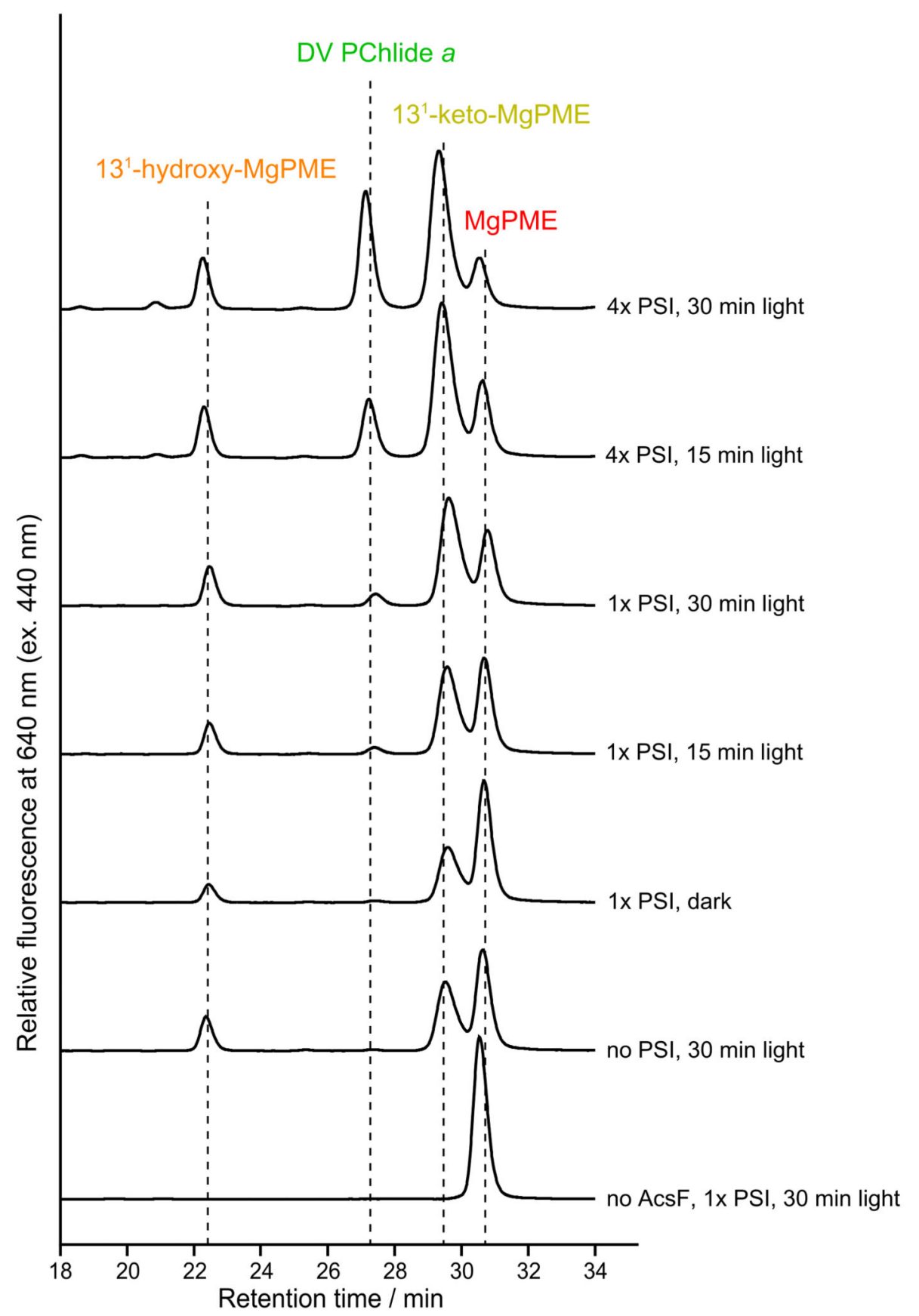

Extended Data Fig. 2. HPLC elution profiles of pigment extracts from coupled PSI-cyclase assays.

A complete assay contained $2 \mu \mathrm{M}$ AcsF, $0.04 \mathrm{mg}$ mL-1 spinach Fd, $14 \mu \mathrm{M}$ MgPME, spinach PSI containing 6 (1x PSI) or $22.4 \mu \mathrm{M}(\sim 4 \mathrm{x}$ PSI) Chl a, $20 \mu \mathrm{M}$ spinach Pc, $2 \mathrm{mM}$ Asc, $60 \mu \mathrm{M}$ DCPIP and $0.29 \mathrm{mg} \mathrm{mL}-1$ catalase. Assays were incubated either in the dark for $30 \mathrm{~min}$, or under red light illumination for 15 or $30 \mathrm{~min}$. Pigment extracts from the assays were analysed by HPLC and pigment elution was monitored by fluorescence at $640 \mathrm{~nm}$ excited at $440 \mathrm{~nm}$. Pigment species were identified by retention times and fluorescence spectra (as in 
Fig. 4). See Supplementary Fig. 4a for HPLC analysis of pigment extracts from additional control assays.

98 EFSGFLIYKLGRRLKNKNPLLAECFNLMSRDEARHAGFLNKAMSDFNLSLDLGFLTKSRKYTFFKPKFIFYATYLSEKIGYWRYITIYRHLEKNPNDCIYPIFEFFENWCQDENRH 214 143 EFSGFLIYKELGRRLKKTNPVVAEIFSLMSRDEARHAGFLNKGLSDFNLALDLGFLTKARKYTFFKPKFIFYATYLSEKIGYWRYITIYRHLKENPEFQCYPIFKYFENWCQDENRH 259 141 EFSGFLIKELARRMKASSPEVAEMFLLMSRDEARHAGFLNKALSDFNLALDLGFLTKNRTYTYFKPKFI IYATFLSEKIGYWRYITIYRHLQRNPDNQFYPLFEYFENWCQDENRH 257 141 EFSGFLIYKELGRRLKATNPVVAEIFTLMSRDEARHAGFLNKAMSDENLALDLGFLTKNRKYTFFKPKFIFYATYLSEKIGYWRYISIYRHLORNPDNQLYPLFEYFENWCQDENRH 257 9 EFSGCVLYNEIQKNV--ENPDVKALMRYMARDESRHAGFINQALRDFGLGIDLGGLKRTKAYTYFKPKYIFYATYLSEKIGYARYITIYRQLERHPDKRFHPIFRWFERWCNDEFRH 213 103 EFSGCVLYKEMKRRG--SNPDIRELFNYMARDEARHAGFINDALREAGVAVNLGFLTKAKKYTYERPKFIYYATYLSEKIGYARYITIYRHLEANPEHRFHPIFKWFKEWCNDEFRH 217

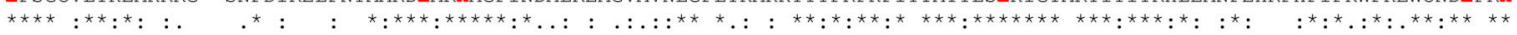

b

\begin{tabular}{|c|c|}
\hline CycI & E- $\mathrm{X}_{31}-\mathrm{EARH}-\mathrm{X}_{41}-\mathbf{E}-\mathrm{X}_{35}-\mathrm{ENRH}$ \\
\hline CHL27 & $E-X_{31}-E A R H-X_{41}-E-X_{35}-E N R H$ \\
\hline CRD1 & E- $X_{31}-E A R H-X_{41}-E-X_{35}-E N R H$ \\
\hline CTH1 & $\mathrm{E}-\mathrm{X}_{31}-\mathrm{EARH}-\mathrm{X}_{41}-\mathrm{E}-\mathrm{X}_{35}-\mathrm{ENRH}$ \\
\hline Acs $F$ & E- $X_{29}-E S R H-X_{41}-E-X_{35}-E F R H$ \\
\hline 0294 & E- $\mathrm{X}_{29}-\mathrm{EARH}-\mathrm{X}_{41}-\mathrm{E}-\mathrm{X}_{35}-\mathrm{EFRH}$ \\
\hline $\mathrm{MMOH}$ & $\mathbf{E}-\mathrm{X}_{29}-\mathbf{E I R H}-\mathrm{X}_{61}-\mathbf{E}-\mathrm{X}_{33}-\mathbf{E L R H}$ \\
\hline
\end{tabular}

C

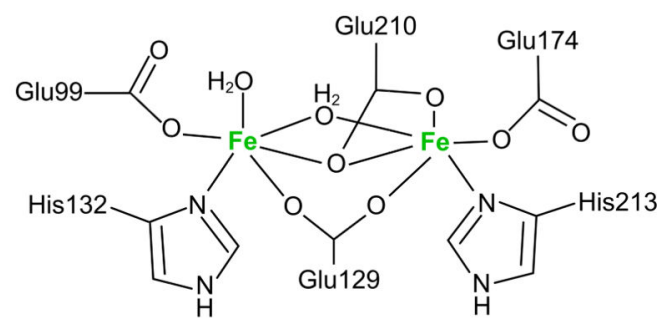

Extended Data Fig. 3. The diiron binding motif and proposed diiron ligation of AcsF.

a, Sequence alignments showing the conserved diiron binding motif of AcsF proteins. Sequences are from Synechocystis sp. PCC 6803 (CycI, BAA16583), Arabidopsis thaliana (CHL27, NP_191253), Chlamydomonas reinhardtii(CRD1, XP_001692557; CTH1, XP_001691047), Rubrivivax gelatinosus IL144 (AcsF, BAL96694) and Rhodobacter sphaeroides 2.4.1 (0294, abbreviated for RSP_0294, YP_353369). Conserved, highly similar and similar residues are marked with asterisks, colons and full stops, respectively. The putative diiron ligands are in red and bold. Full-length protein sequences were used for alignments but for clarity, only the putative diiron binding motifs with the residue range indicated, are shown. b, Sequence homologies between the diiron binding motifs of AcsF proteins and the soluble methane monooxygenase hydroxylase subunit from Methylococcus capsulatus Bath (MMOH, P22869). c, Proposed coordination of the diiron ligands of AcsF at the diferrous state based on the crystal structure of MMOH (PDB, 1FYZ)51.

\section{Supplementary Material}

Refer to Web version on PubMed Central for supplementary material.

\section{Acknowledgements}

We thank Dr David Swainsbury, University of Sheffield, for providing membrane protein standards for the gel filtration calibration and Dr Nathan Soulier, The Pennsylvania State University, for supplying the BL21(DE3) $\Delta i s c R$ strain. G.E.C., N.B.P.A. P.J.J. and C.N.H. gratefully acknowledge financial support from the Biotechnology and Biological Sciences Research Council (BBSRC U.K.), award number BB/M000265/1. C.N.H. is also supported by European Research Council Synergy Award 854126. M.J.D. was supported by BBSRC U.K., award number BB/ M012166/1.

\section{Data availability}

All supporting data are included in the Supplementary Information. Annotated source data are provided with this paper for Figs. 2b, 2c, 2e, 4, Extended Data Figs. 1, 2, and 
Supplementary Figs. 1, 4a, 4b. The mass spectrometry raw data-files used for Fig. 5 and Supplementary Fig. 3 have been deposited to https://figshare.shef.ac.uk/articles/dataset/ 13655774.

\section{Code availability}

No custom code was used for the study.

\section{References}

1. Porra RJ, Schäfer W, Gad'on N, Katheder I, Drews G, Scheer H. Origin of the two carbonyl oxygens of bacteriochlorophyll a Demonstration of two different pathways for the formation of ring $\mathrm{E}$ in Rhodobacter sphaeroides and Roseobacter denitrificans and a common hydratase mechanism for 3acetyl group formation. Eur J Biochem. 1996; 239:85-92. [PubMed: 8706723]

2. Porra RJ, Urzinger M, Winkler J, Bubenzer C, Scheer H. Biosynthesis of the 3 -acetyl and $13^{1}$-oxo groups of bacteriochlorophyll $a$ in the facultative aerobic bacterium, Rhodovulum sulfidophilum: The presence of both oxygenase and hydratase pathways for isocyclic ring formation. Eur $\mathrm{J}$ Biochem. 1998; 257:185-191. [PubMed: 9799118]

3. Wiesselmann M, Hebecker S, Borrero-de Acuña JM, Nimtz M, Bollivar D, Jänsch L, Moser J, Jahn D. Mg-protoporphyrin IX monomethyl ester cyclase from Rhodobacter capsulatus: Radical SAMdependent synthesis of the isocyclic ring of bacteriochlorophylls. Biochem J. 2020

4. Chen GE, Canniffe DP, Hunter CN. Three classes of oxygen-dependent cyclase involved in chlorophyll and bacteriochlorophyll biosynthesis. Proc Natl Acad Sci USA. 2017; 114:6280-6285. [PubMed: 28559347]

5. Pinta V, Picaud M, Reiss-Husson F, Astier C. Rubrivivax gelatinosus acsF (previously orf358) codes for a conserved, putative binuclear-iron-cluster-containing protein involved in aerobic oxidative cyclization of Mg-protoporphyrin IX monomethylester. J Bacteriol. 2002; 184:746-753. [PubMed: 11790744]

6. Hollingshead S, Kopečá J, Jackson PJ, Canniffe DP, Davison PA, Dickman MJ, Sobotka R, Hunter $\mathrm{CN}$. Conserved chloroplast open-reading frame $y c f 54$ is required for activity of the magnesium protoporphyrin monomethylester oxidative cyclase in Synechocystis PCC 6803. J Biol Chem. 2012; 287:27823-27833. [PubMed: 22711541]

7. Albus CA, Salinas A, Czarnecki O, Kahlau S, Rothbart M, Thiele W, Lein W, Bock R, Grimm B, Schottler MA. LCAA a novel factor required for magnesium protoporphyrin monomethylester cyclase accumulation and feedback control of aminolevulinic acid biosynthesis in tobacco. Plant Physiol. 2012; 160:1923-1939. [PubMed: 23085838]

8. Granick S. The structural and functional relationships between heme and chlorophyll. Harvey Lect Series. 1950; 44:220-245.

9. Chereskin BM, Castelfranco PA. Effects of iron and oxygen on chlorophyll biosynthesis : II. Observations on the biosynthetic pathway in isolated etiochloroplasts. Plant Physiol. 1982; 69:112116. [PubMed: 16662140]

10. Chereskin BM, Wong YS, Castelfranco PA. In vitro synthesis of the chlorophyll isocyclic ring: Transformation of magnesium-protoporphyrin IX and magnesium-protoporphyrin IX monomethyl ester into magnesium-2,4-divinyl pheoporphyrin a5 . Plant Physiol. 1982; 70:987-993. [PubMed: 16662656]

11. Nasrulhaq-Boyce A, Griffiths WT, Jones OT. The use of continuous assays to characterize the oxidative cyclase that synthesizes the chlorophyll isocyclic ring. Biochem J. 1987; 243:23-29. [PubMed: 3606572]

12. Walker CJ, Mansfield KE, Smith KM, Castelfranco PA. Incorporation of atmospheric oxygen into the carbonyl functionality of the protochlorophyllide isocyclic ring. Biochem J. 1989; 257:599602. [PubMed: 2930469] 
13. Wong YS, Castelfranco PA. Resolution and reconstitution of Mg-protoporphyrin IX monomethyl ester (oxidative) cyclase, the enzyme system responsible for the formation of the chlorophyll isocyclic ring. Plant Physiol. 1984; 75:658-661. [PubMed: 16663682]

14. Bollivar DW, Beale SI. Formation of the isocyclic ring of chlorophyll by isolated Chlamydomonas reinhardtii chloroplasts. Photosynth Res. 1995; 43:113-124. [PubMed: 24306744]

15. Bollivar DW, Beale SI. The chlorophyll biosynthetic enzyme Mg-protoporphyrin IX monomethyl ester (oxidative) cyclase: Characterization and partial purification from Chlamydomonas reinhardtii and Synechocystis sp. PCC 6803. Plant Physiol. 1996; 112:105-114. [PubMed: 12226378]

16. Rzeznicka K, Walker CJ, Westergren T, Kannangara CG, von Wettstein D, Merchant S, Gough SP, Hansson M. Xantha-I encodes a membrane subunit of the aerobic Mg-protoporphyrin IX monomethyl ester cyclase involved in chlorophyll biosynthesis. Proc Natl Acad Sci USA. 2005; 102:5886-5891. [PubMed: 15824317]

17. Ellsworth RK, Aronoff S. Investigations on the biogenesis of chlorophyll $a$ : III. Biosynthesis of Mg-vinylpheoporphine a 5 methylester from Mg-protoporphine IX monomethylester as observed in Chlorella mutants. Arch Biochem Biophys. 1968; 125:269-277. [PubMed: 5649520]

18. Ellsworth RK, Aronoff S. Investigations of the biogenesis of chlorophyll a: IV. Isolation and partial characterization of some biosynthetic intermediates between Mg-protoporphine IX monomethyl ester and Mg-vinylpheoporphine a 5, obtained from Chlorella mutants. Arch Biochem Biophys. 1969; 130:374-383. [PubMed: 5778652]

19. Wong YS, Castelfranco PA. Properties of the Mg-protoporphyrin IX monomethyl ester (oxidative) cyclase system. Plant Physiol. 1985; 79:730-733. [PubMed: 16664482]

20. Walker CJ, Mansfield KE, Rezzano IN, Hanamoto CM, Smith KM, Castelfranco PA. The magnesium-protoporphyrin IX (oxidative) cyclase system: Studies on the mechanism and specificity of the reaction sequence. Biochem J. 1988; 255:685-692. [PubMed: 3202840]

21. Chen GE, Canniffe DP, Barnett SFH, Hollingshead S, Brindley AA, Vasilev C, Bryant DA, Hunter CN. Complete enzyme set for chlorophyll biosynthesis in Escherichia coli . Sci Adv. 2018; 4:eaaq1407. [PubMed: 29387799]

22. Miroux B, Walker JE. Over-production of proteins in Escherichia coli: Mutant hosts that allow synthesis of some membrane proteins and globular proteins at high levels. J Mol Biol. 1996; 260:289-298. [PubMed: 8757792]

23. Strop P, Brunger AT. Refractive index-based determination of detergent concentration and its application to the study of membrane proteins. Protein Sci. 2005; 14:2207-2211. [PubMed: 16046633]

24. Fox BG, Shanklin J, Somerville C, Münck E. Stearoyl-acyl carrier protein $\Delta^{9}$ desaturase from Ricinus communis is a diiron-oxo protein. Proc Natl Acad Sci USA. 1993; 90:2486-2490. [PubMed: 8460163]

25. Makris TM, Chakrabarti M, Münck E, Lipscomb JD. A family of diiron monooxygenases catalyzing amino acid beta-hydroxylation in antibiotic biosynthesis. Proc Natl Acad Sci USA. 2010; 107:15391-15396. [PubMed: 20713732]

26. Behan RK, Lippard SJ. The aging-associated enzyme CLK-1 is a member of the carboxylatebridged diiron family of proteins. Biochemistry. 2010; 49:9679-9681. [PubMed: 20923139]

27. Shanklin J, Somerville C. Stearoyl-acyl-carrier-protein desaturase from higher plants is structurally unrelated to the animal and fungal homologs. Proc Natl Acad Sci USA. 1991; 88:2510-2514. [PubMed: 2006187]

28. Choi YS, Zhang H, Brunzelle JS, Nair SK, Zhao H. In vitro reconstitution and crystal structure of p-aminobenzoate $\mathrm{N}$-oxygenase (AurF) involved in aureothin biosynthesis. Proc Natl Acad Sci USA. 2008; 105:6858-6863. [PubMed: 18458342]

29. Helfrich M, Ross A, King GC, Turner AG, Larkum AWD. Identification of [8-vinyl]protochlorophyllide $a$ in phototrophic prokaryotes and algae: chemical and spectroscopic properties. Biochim Biophys Acta. 1999; 1410:262-272. [PubMed: 10082792]

30. Klement H, Helfrich M, Oster U, Schoch S, Rüdiger W. Pigment-free NADPH:protochlorophyllide oxidoreductase from Avena sativa L: Purificaton and substrate specificity. Eur J Biochem. 1999; 265:862-874. [PubMed: 10518779] 
31. Karger GA, Reid JD, Hunter CN. Characterization of the binding of deuteroporphyrin IX to the magnesium chelatase $\mathrm{H}$ subunit and spectroscopic properties of the complex. Biochemistry. 2001; 40:9291-9299. [PubMed: 11478896]

32. Meneely KM, Sundlov JA, Gulick AM, Moran GR, Lamb AL. An open and shut case: the interaction of magnesium with MST enzymes. J Am Chem Soc. 2016; 138:9277-9293. [PubMed: 27373320]

33. Schäfer M, Drayß M, Springer A, Zacharias P, Meerholz K. Radical cations in electrospray mass spectrometry: formation of open-shell species, examination of the fragmentation behaviour in ESI$\mathrm{MS}^{\mathrm{n}}$ and reaction mechanism studies by detection of transient radical cations. Eur J Org Chem. 2007; 2007:5162-5174.

34. Jensen K, Johnston JB, de Montellano PRO, Møller BL. Photosystem I from plants as a bacterial cytochrome P450 surrogate electron donor: terminal hydroxylation of branched hydrocarbon chains. Biotechnol Lett. 2012; 34:239-245. [PubMed: 21983973]

35. Biegel E, Schmidt S, González JM, Müller V. Biochemistry, evolution and physiological function of the Rnf complex, a novel ion-motive electron transport complex in prokaryotes. Cell Mol Life Sci. 2011; 68:613-634. [PubMed: 21072677]

36. Buckel W, Thauer RK. Energy conservation via electron bifurcating ferredoxin reduction and proton/ $\mathrm{Na}^{+}$translocating ferredoxin oxidation. Biochim Biophys Acta. 2013; 1827:94-113. [PubMed: 22800682]

37. Chen GE, Hunter CN. Protochlorophyllide synthesis by recombinant cyclases from eukaryotic oxygenic phototrophs and the dependence on Ycf54. Biochem J. 2020; 477:2313-2325. [PubMed: 32469391]

38. Herbst J, Girke A, Hajirezaei MR, Hanke G, Grimm B. Potential roles of YCF54 and ferredoxinNADPH reductase for magnesium protoporphyrin monomethylester cyclase. Plant J. 2018; 94:485-496. [PubMed: 29443418]

39. Stuart D, Sandström M, Youssef HM, Zakhrabekova S, Jensen PE, Bollivar DW, Hansson M. Aerobic barley Mg-protoporphyrin IX monomethyl ester cyclase is powered by electrons from ferredoxin. Plants. 2020; 9:1157.

40. Steccanella V, Hansson M, Jensen PE. Linking chlorophyll biosynthesis to a dynamic plastoquinone pool. Plant Physiol Biochem. 2015; 97:207-216. [PubMed: 26480470]

41. Lea-Smith DJ, Bombelli P, Vasudevan R, Howe CJ. Photosynthetic, respiratory and extracellular electron transport pathways in cyanobacteria. Biochim Biophys Acta. 2016; 1857:247-255. [PubMed: 26498190]

42. Heyes DJ, Martin GE, Reid RJ, Hunter CN, Wilks HM. NADPH:protochlorophyllide oxidoreductase from Synechocystis: overexpression, purification and preliminary characterisation. FEBS Lett. 2000; 483:47-51. [PubMed: 11033354]

43. Reid JD, Hunter CN. Magnesium-dependent ATPase activity and cooperativity of magnesium chelatase from Synechocystis sp. PCC6803. J Biol Chem. 2004; 279:26893-26899. [PubMed: 15051720]

44. Shepherd M, Hunter CN. Transient kinetics of the reaction catalysed by magnesium protoporphyrin IX methyltransferase. Biochem J. 2004; 382:1009-1013. [PubMed: 15239672]

45. Shepherd M, Reid JD, Hunter CN. Purification and kinetic characterization of the magnesium protoporphyrin IX methyltransferase from Synechocystis PCC6803. Biochem J. 2003; 371:351360. [PubMed: 12489983]

46. Wong YS, Castelfranco PA, Goff DA, Smith KM. Intermediates in the formation of the chlorophyll isocyclic ring. Plant Physiol. 1985; 79:725-729. [PubMed: 16664481]

47. Rebeiz CA, Mattheis JR, Smith BB, Rebeiz CC, Dayton DF. Chloroplast biogenesis: Biosynthesis and accumulation of $\mathrm{Mg}$-protoporphyrin IX monoester and longer wavelength metalloporphyrins by greening cotyledons. Arch Biochem Biophys. 1975; 166:446-465. [PubMed: 1119802]

48. Walters KA, Golbeck JH. Designing a modified clostridial 2[4Fe-4S] ferredoxin as a redox coupler to directly link photosystem I with a Pt nanoparticle. Photosynth Res. 2020; 143:165-181.

[PubMed: 31643016] 
49. Artimo P, Jonnalagedda M, Arnold K, Baratin D, Csardi G, de Castro E, Duvaud S, Flegel V, Fortier A, Gasteiger E, Grosdidier A, et al. ExPASy: SIB bioinformatics resource portal. Nucleic Acids Res. 2012; 40:W597-W603. [PubMed: 22661580]

50. Pueyo JJ, Gómez-Moreno C. Purification of ferredoxin-NADP ${ }^{+}$reductase, flavodoxin and ferredoxin from a single batch of the cyanobacterium Anabaena PCC 7119. Prep Biochem. 1991; 21:191-204. [PubMed: 1664099]

51. Whittington DA, Lippard SJ. Crystal structures of the soluble methane monooxygenase hydroxylase from Methylococcus capsulatus (Bath) demonstrating geometrical variability at the dinuclear iron active site. J Am Chem Soc. 2001; 123:827-838. [PubMed: 11456616] 


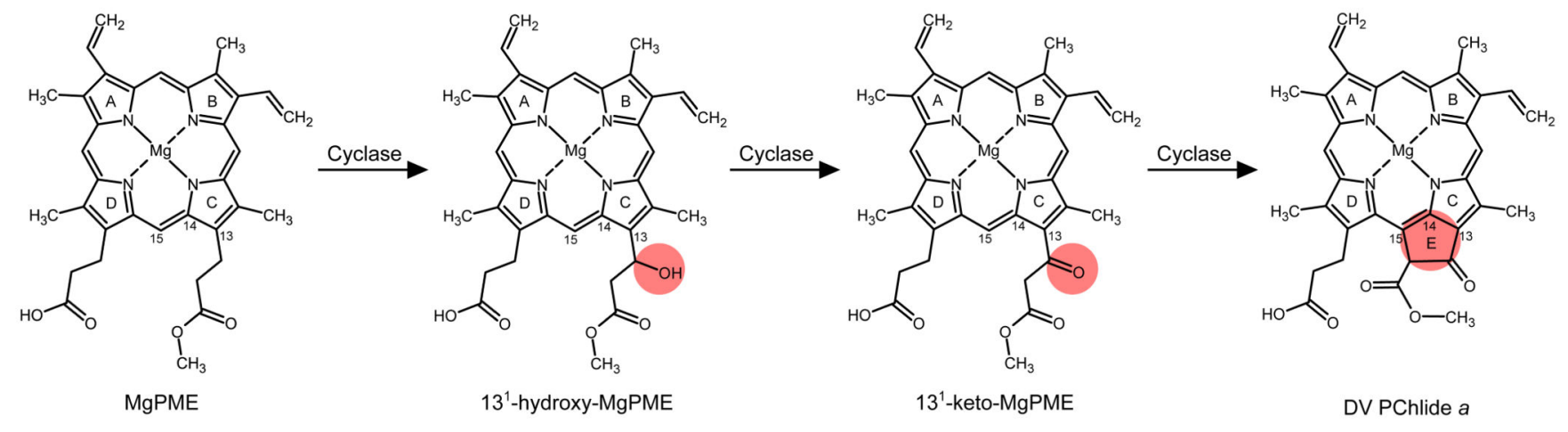

Fig. 1. Proposed reaction intermediates of MgPME cyclase.

Formation of the $\mathrm{E}$ ring of chlorophyll is proposed to proceed via hydroxylation, oxidation and cyclisation of the $\mathrm{C} 13$ methylpropionyl side chain of MgPME. The chemical change at each step is highlighted. International Union of Pure and Applied Chemistry numbering of the relevant macrocycle carbons is indicated. 

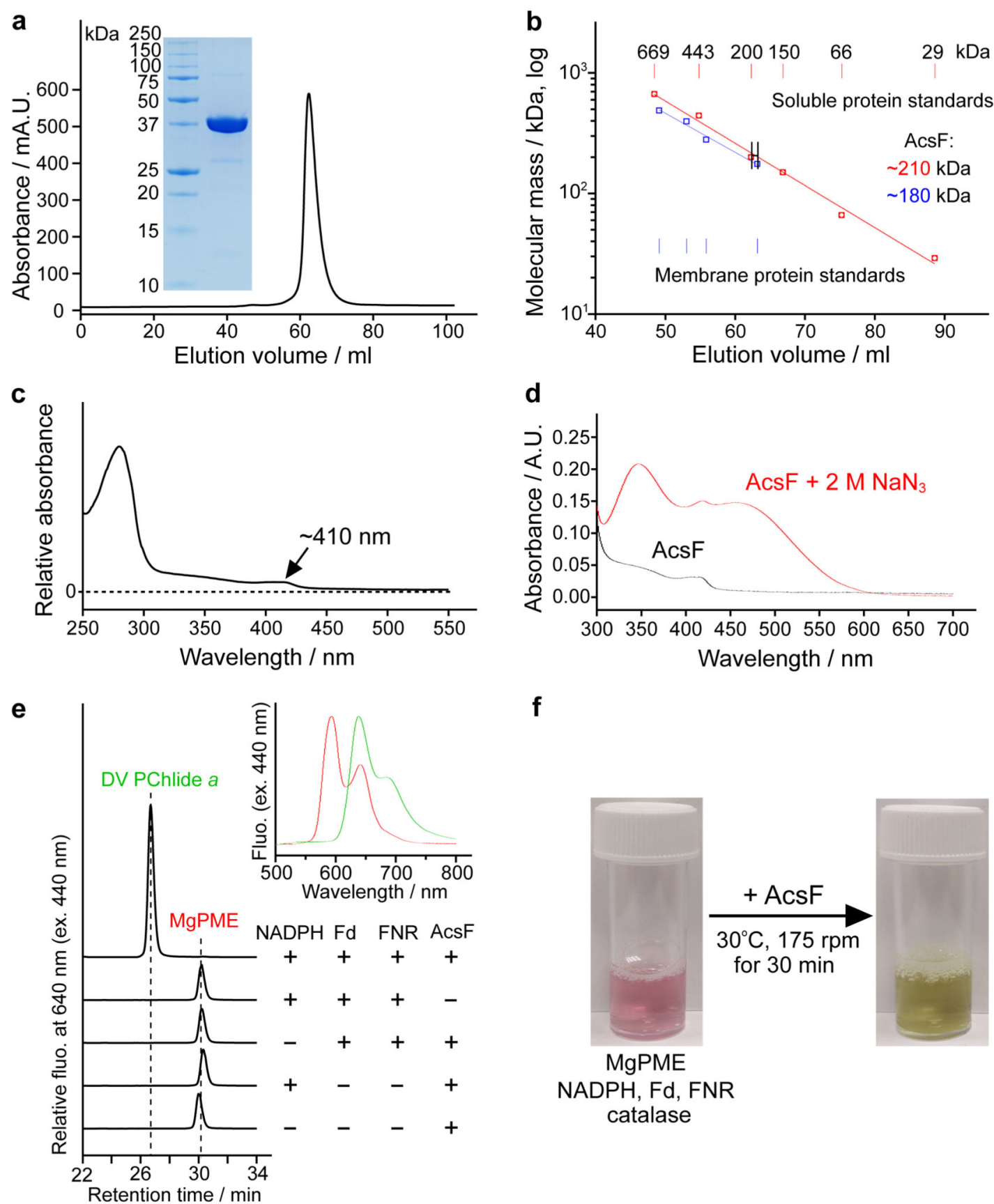

f

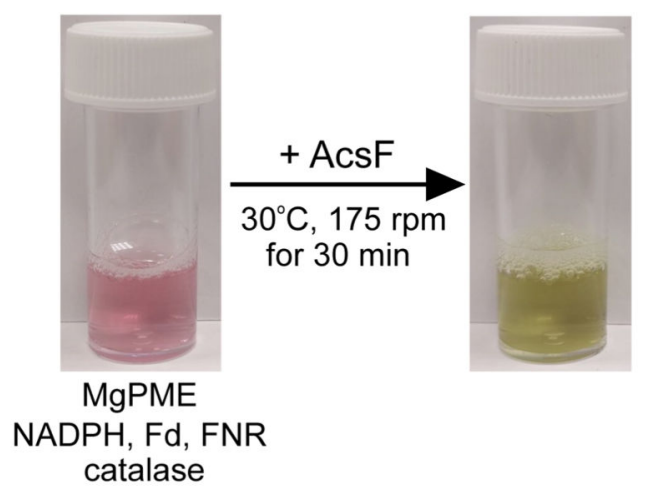

Fig. 2. Purification, spectral characterisation and reconstitution of cyclase activity of AcsF. a, Gel filtration profile of AcsF on a HiLoad 16/600 Superdex 200 prep grade column monitored by absorbance at $280 \mathrm{~nm}$ and SDS-PAGE analysis of $10 \mu \mathrm{g}$ purified AcsF (inset). Shown are representative of three independently repeated experiments. b, Estimate of the molecular mass of native AcsF from triplicate gel filtration runs (range of elution volume indicated) using calibration curves (logarithm of molecular mass vs elution volume) generated from the data points of soluble (red circles) and membrane (blue squares) protein standards using nonlinear regression analysis. Membrane protein standards of 104, 208, 325 
and $416 \mathrm{kDa}$ were used and a value of $72 \mathrm{kDa}$ (size of $\beta$-DDM micelles) was added to each molecular mass when generating the calibration curve. The calculated molecular mass values (inset) include the contribution of bound $\beta$-DDM molecules. c, Absorbance spectrum of AcsF as isolated. d, Absorbance spectra of $8 \mu \mathrm{M}$ AcsF in the absence and presence of 2 $\mathrm{M}$ sodium azide. $\mathbf{e}$, Cofactor requirements for in vitro cyclase activity of AcsF revealed by end-point HPLC-based assays. A complete assay contained 3.7 $\mu \mathrm{M}$ AcsF, $10 \mu \mathrm{M}$ MgPME, 2 mM NADPH, $0.2 \mathrm{mg} \mathrm{mL}^{-1}$ spinach Fd, 0.4 units $\mathrm{mL}^{-1}$ spinach FNR and $0.29 \mathrm{mg} \mathrm{mL}^{-1}$ catalase. Retention times and fluorescence spectra (inset) were used to identify pigment species. See Materials and Methods for experimental details. f, Photographs showing the marked colour change showing the activity of AcsF in an assay containing $27 \mu \mathrm{M} \mathrm{MgPME}$ and other assay components at the same concentration as in $\mathbf{a}$. 


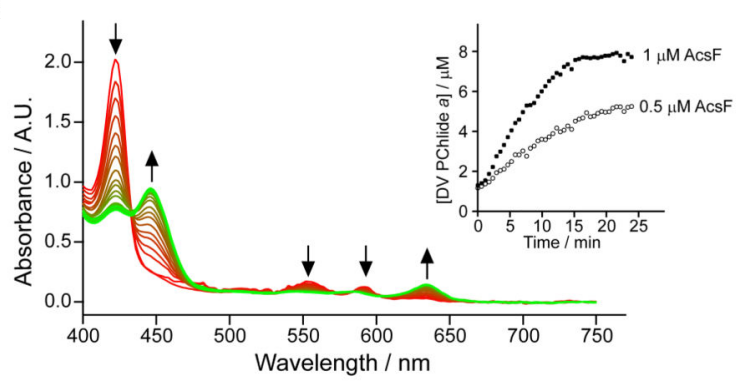

d

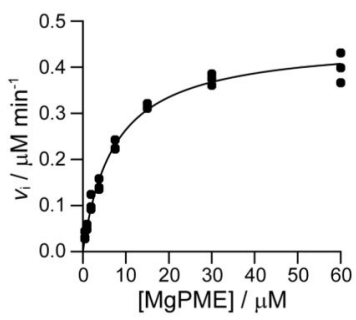

b

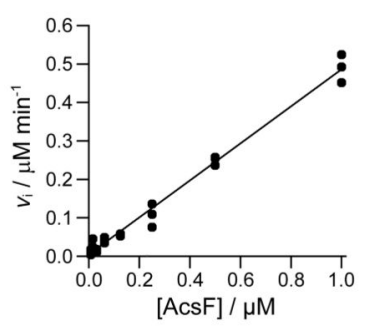

$\mathbf{f}$

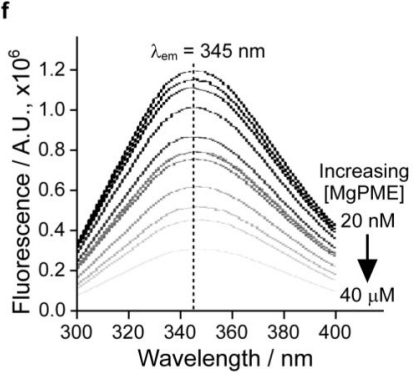

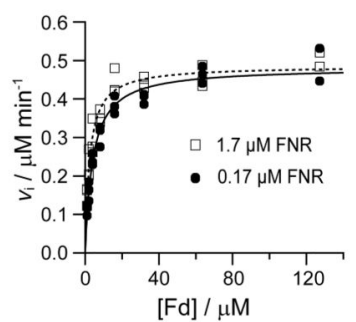

g

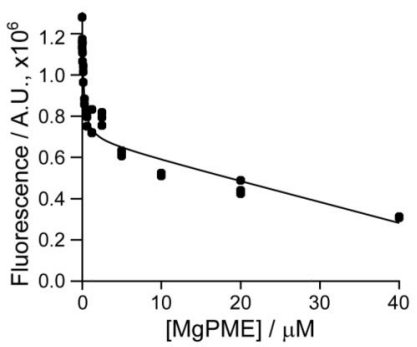

Fig. 3. Steady-state kinetics of AcsF, and binding of MgPME to AcsF analysed by tryptophan fluorescence quenching.

a, The progressive spectral change during a continuous absorbance-based cyclase assay, which contained $1 \mu \mathrm{M}$ AcsF, $10 \mu \mathrm{M}$ MgPME, $7.6 \mu \mathrm{M}$ Anabaena Fd, $0.17 \mu \mathrm{M}$ Anabaena FNR, $2.5 \mathrm{mM}$ NADPH and $0.29 \mathrm{mg} \mathrm{mL}^{-1}$ catalase. Arrows indicate the direction of change. Inset shows the product (DV PChlide a) evolution with 0.5 and $1 \mu \mathrm{M}$ AcsF, monitored by absorbance at $634 \mathrm{~nm}$. b-e, The dependence of the initial rate of product formation on AcsF (b), Anabaena Fd (c), MgPME (d) and NADPH (e). Assay conditions were as in a except the following stated differences: $\mathbf{b}, 7.81 \mathrm{nM}$ to $1 \mu \mathrm{M}$ AcsF; $\mathbf{c}, 0.5 \mu \mathrm{M}$ AcsF, 0.17 or $1.7 \mu \mathrm{M}$ Anabaena FNR, 0.99 to $127 \mu \mathrm{M}$ Anabaena Fd; d, $0.5 \mu \mathrm{M}$ AcsF, $1.7 \mu \mathrm{M}$ Anabaena FNR, 31 $\mu \mathrm{M}$ Anabaena Fd; e, $0.5 \mu \mathrm{M}$ AcsF, $30 \mu \mathrm{M}$ MgPME, $1.7 \mu \mathrm{M}$ Anabaena FNR, $31 \mu \mathrm{M}$ Anabaena Fd, $62.5 \mu \mathrm{M}$ to $4 \mathrm{mM}$ NADPH. Each data point is an independent experiment. The Michaelis-Menten equation (equation 1, see Materials and Methods) was fitted to the kinetic data in $\mathbf{c}$ and $\mathbf{d}$, with the characterising parameters $K_{\mathrm{M}}$ (apparent) $=4.05 \pm 0.39 \mu \mathrm{M}$ $(0.17 \mu \mathrm{M}$ FNR $)$ or $2.41 \pm 0.26 \mu \mathrm{M}(1.7 \mu \mathrm{M}$ FNR $)(\mathbf{c}) ;=0.91 \pm 0.02 \mathrm{~min}^{-1},=7.03 \pm 0.51 \mu \mathrm{M}$ (d). The Hill equation (equation 2, see Materials and Methods) was fitted to the NADPH titration data with $=1.06 \pm 0.01 \mathrm{~min}^{-1},=0.16 \pm 0.01 \mathrm{mM}, n=2.1 \pm 0.1$ (e). f, A series of spectra showing quenching of AcsF fluorescence by MgPME. Excitation was set at $280 \mathrm{~nm}$, producing an emission maximum at $345 \mathrm{~nm}$. The average fluorescence spectra of triplicate experiments are shown. g, Plot of AcsF fluorescence against MgPME concentration. Each data point is an independent experiment. The curve fit is described by a modified single-site binding model (equation 3, see Materials and Methods) with $K_{\mathrm{d}}$ for MgPME binding of $0.16 \pm 0.05 \mu \mathrm{M}$. 


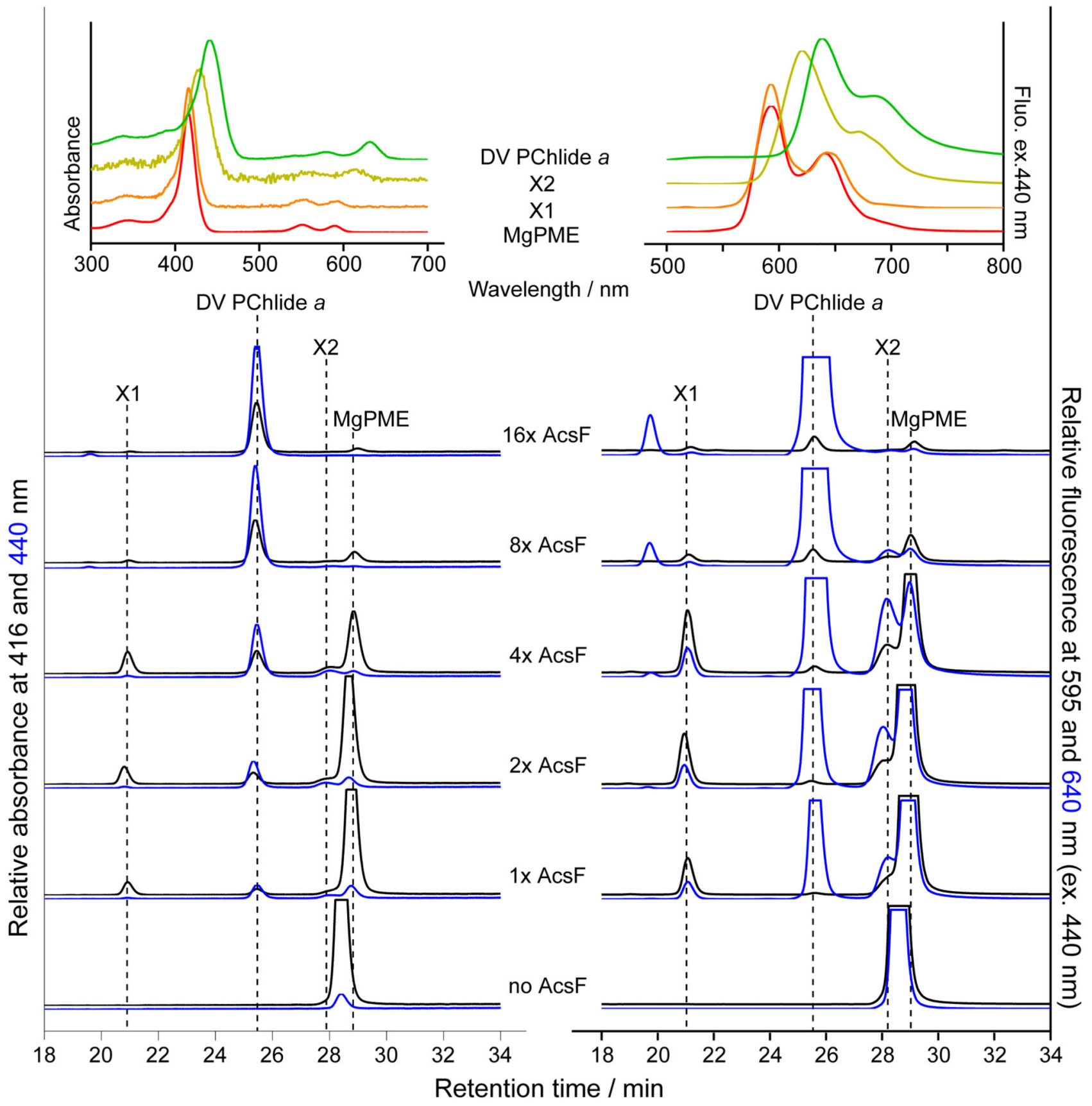

Fig. 4. HPLC elution profiles of pigment extracts from end-point cyclase assays at various AcsF concentrations.

Assay conditions were $20 \mu \mathrm{M}$ MgPME, $0.2 \mathrm{mg} \mathrm{mL}^{-1}$ spinach $\mathrm{Fd}, 0.4$ units $\mathrm{mL}^{-1}$ spinach FNR, 2 mM NADPH, $0.29 \mathrm{mg} \mathrm{mL}^{-1}$ catalase and AcsF at various concentrations from 0.23 (1x AcsF) to 3.68 (16x AcsF) $\mu \mathrm{M}$. Assays were initiated by adding AcsF and terminated after 30 min incubation. Pigment extracts from the assays were analysed by HPLC with elution of pigment species monitored by absorbance at 416 and $440 \mathrm{~nm}$ and by fluorescence at 595 and $640 \mathrm{~nm}$ with excitation at $440 \mathrm{~nm}$. Insets show the acquired absorbance and 
fluorescence spectra of MgPME, DV PChlide a, and the potential reaction intermediates, X1 and X2. 

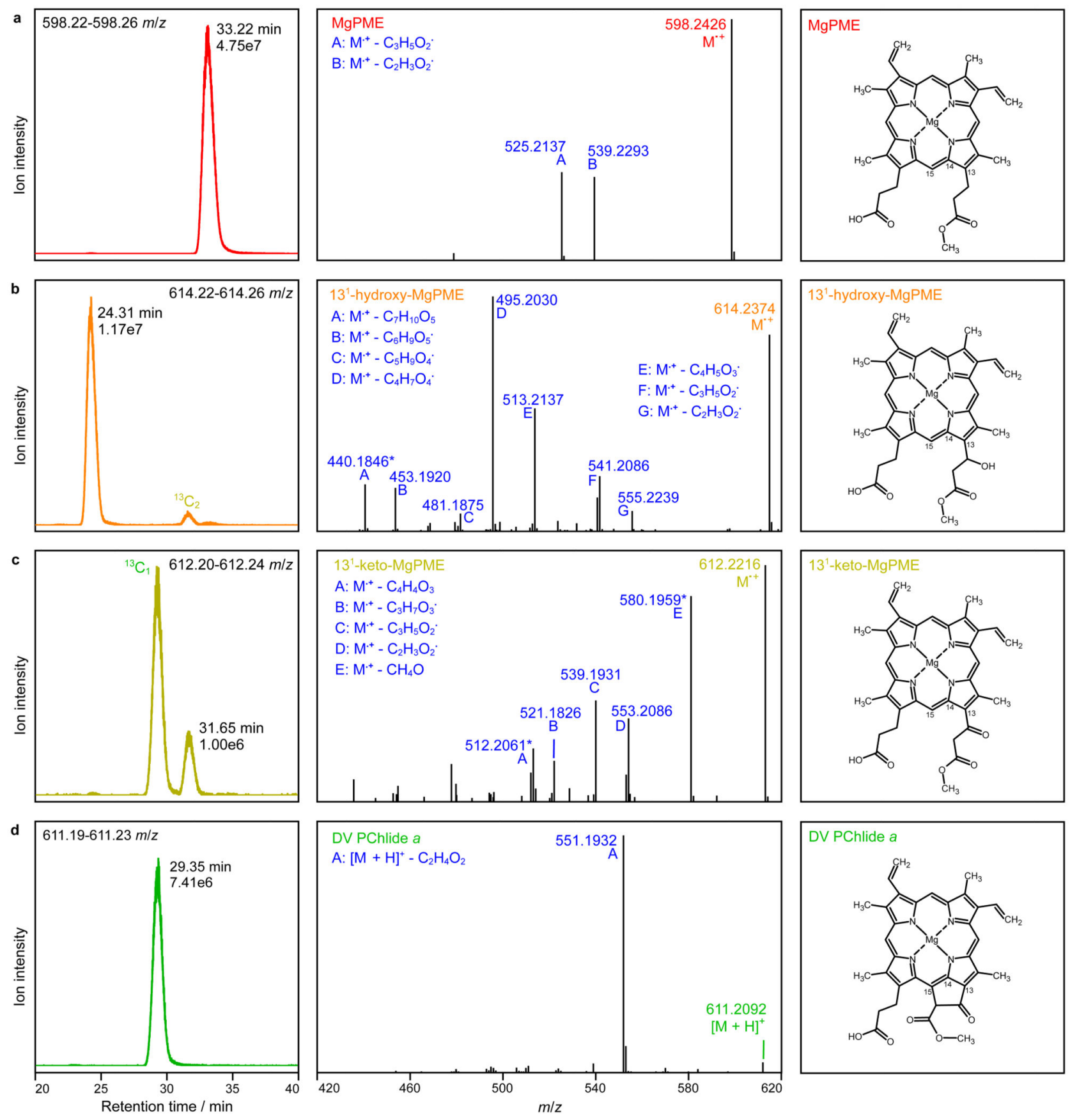

DV PChlide

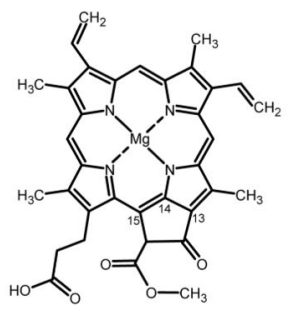

Fig. 5. Analysis of extracted pigments by LC-ESI-MS/MS.

The pigment extract from scaled-up in vitro cyclase assays corresponding to $4 \mathrm{x} A \operatorname{csF}(0.92$ $\mu \mathrm{M}$ ) in Fig. 4 was analysed. Extracted ion chromatograms (EICs) and product ion spectra derived from HCD of selected monoisotopic molecular ions are shown in the left and centre panels, respectively: a, MgPME, b, $13^{1}$-hydroxy-MgPME, c, $13^{1}$-keto-MgPME, d, DV PChlide $a$. The molecular structures that align with the mass spectral evidence presented here are shown in the corresponding right panels. EICs were generated for the indicated $\mathrm{m} / z$ ranges covering the target monoisotopic ions with peaks labelled with their retention times 
and ion intensities. Peaks mapping to ${ }^{13} \mathrm{C}$-containing isotopomers that fall within the EIC range are also labelled. Cations generated by gas phase neutral loss reactions are indicated by upper case letters with the eliminated molecular formulas also listed. The majority of product ions are carbocations formed after radical neutral loss; those labelled with an asterisk are radical cations formed after even electron neutral loss. Details of the structures which validate the identifications of the cyclase substrate, intermediates and product are shown in Supplementary Fig. 3. 

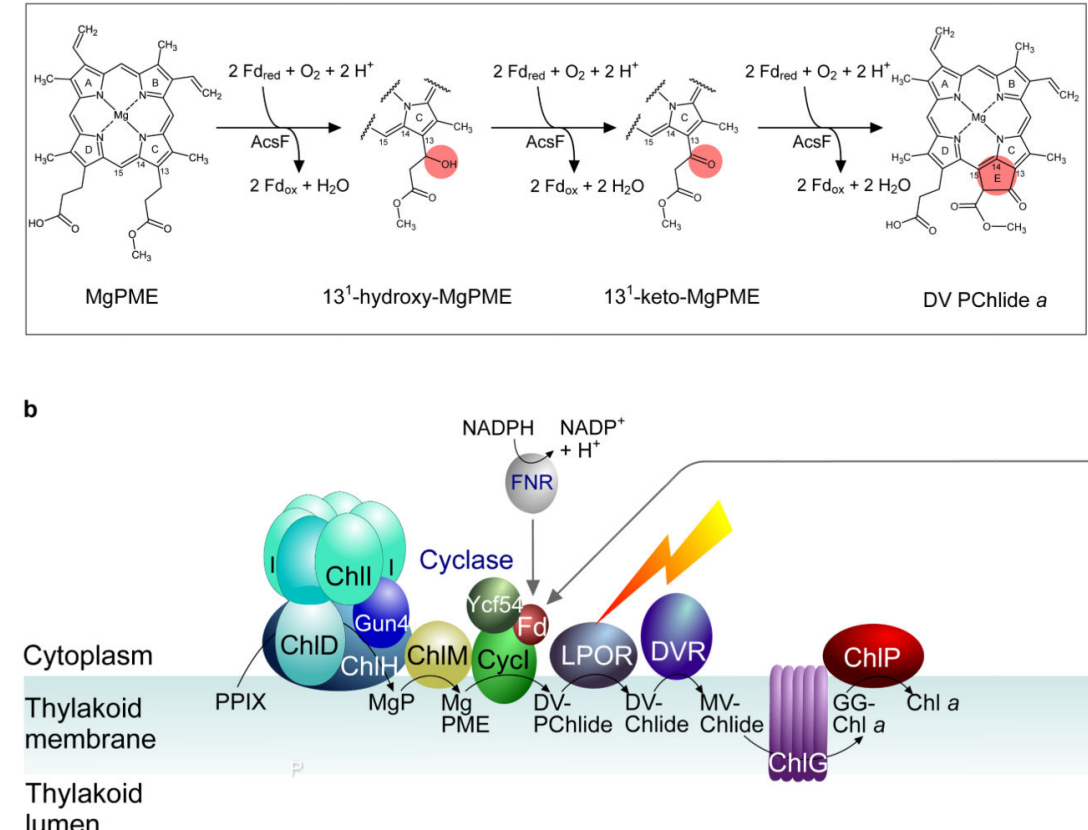

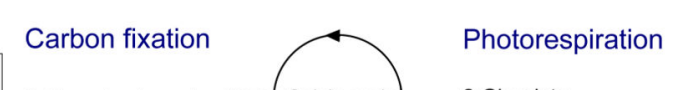

Carbon fixation

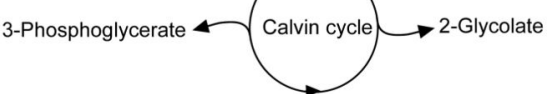

ChIP

GG- Chl a Chl a

Fig. 6. Diagram depicting the Fd-dependent cyclase reaction catalysed by AcsF and the supply of reduced Fd, directly or indirectly, by PSI.

a, The updated sequence of cyclase reactions catalysed by AcsF, with the chemical change of the porphyrin substrate at each step highlighted in the pink circle. $\mathrm{Fd}_{\mathrm{red}}$ and $\mathrm{Fd}_{\mathrm{ox}}$ represent reduced and oxidised Fd, respectively. b. The chlorophyll biosynthesis pathway is shown on the left, progressing from protoporphyrin IX (PPIX) to chlorophyll a (Chl a), via magnesium protoporphyrin IX (MgP), Mg-protoporphyrin IX monomethyl ester (MgPME), 3,8-divinyl protochlorophyllide a (DV-PChlide), divinyl chlorophyllide $a$ (DV-Chlide), monovinyl chlorophyllide $a$ (MV-Chlide), and geranylgeranyl-chlorophyll $a$ (GG-Chl $a$ ). $\mathrm{ChlH}, \mathrm{D}, \mathrm{I}$ are subunits, and Gun4 is an accessory protein, of the magnesium chelatase complex; ChlM is the MgP methyltransferase; CycI is the counterpart of the AcsF cyclase in cyanobacteria and plants, shown here with the accessory protein Ycf54 and Fd; LPOR is the light-dependent PChlide oxidoreductase; DVR is the divinyl reductase, the cyanobacterial version (BciB) of which requires Fd, whereas the plant DVR does not. ChlG is the chlorophyll synthase and ChlP is the geranylgeranyl reductase. The diagram (right) depicts a possible direct link between PSI and chlorophyll biosynthesis, showing that PSI could provide reduced Fd for the cyclase reaction; FNR-based reduction of Fd is also depicted, corresponding to the in vitro assays in Fig. 3 and Extended Data Fig. 2 respectively. Reduced Fd also provides electrons for a variety of cellular functions, shown here for cyanobacterial metabolism and adapted from the diagram in ref. ${ }^{41}$. PcyA, phycocyanobilin:Fd oxidoreductase; GlsF, Fd-dependent glutamate synthase; FtrC/V, Fd:thioredoxin reductase; FNR, Fd:NADP ${ }^{+}$reductase; Sir, Fd:sulfite reductase; NarB, nitrate reductase; NirA, nitrite reductase; Flv1/3, Flavodiiron 1/3; HOX, bi-directional hydrogenase. 